\title{
3. Queere Bewegungsgeschichte
}

Obwohl im Zentrum der vorliegenden Untersuchung der queere Aktivismus im deutschsprachigen Raum steht, beginnt dessen bewegungsgeschichtliche Situierung in den USA. Zum einen, weil die im vorherigen Abschnitt ausgeführten Theoretisierungen queeren Widerstands unter anderem vom queeren US-amerikanischen Aktivismus ausgehen. Zum anderen ist "queer « im deutschsprachigen Raum aus dem US-Amerikanischen angeeignet worden. ${ }^{1}$ Bis heute nehmen aktuelle queere Politiken aus dem deutschsprachigen Raum - so auch die für die empirische Untersuchung ausgewählten queeren Projekte - Bezug auf die US-amerikanische queere Bewegungsgeschichte, ebenso wie auf die aus ihr heraus formulierten Theorien. ${ }^{2}$ Den Ausführungen zu der Entstehung queerer Politiken in den USA folgt anschließend eine Bewegungsgeschichte queerer Politiken in Österreich, Deutschland und der Schweiz.

\subsection{Der US-amerikanische Beginn}

Als »Moment der Artikulation von queer« gilt zumeist die Aids-Krise der 1980er-Jahre, in der sich queere Politiken in Abgrenzung zu schwul-lesbischen Identitätspolitiken etabliert haben. ${ }^{3}$ Aber auch vor der Aids-Krise hat es Kämpfe und Bewegungen gegeben, die nicht nur schwul-lesbisch waren. Sie wurden aber im Nachhinein häufig als solche erinnert. Mittlerweile gibt es viele Versuche, diese Erinnerungskulturen $\mathrm{zu}$ »queeren« (durchkreuzen) und dadurch »queer« in die Prä-Aids-Bewegungsgeschichte einzuschreiben. Zentral sind dabei die Art und Weise, wie das diskursive Ereignis der "Stonewall Inn«-Proteste 1969 erinnert wird. Entsprechend beginnt diese queere Bewegungsgeschichte nicht mit der Aids-Krise, sondern mit den diskursiven Kämpfen um das Ereignis "Stonewall«, die nicht zuletzt auch deswegen immer noch diskutiert

Annamarie Jagose: Queer Theory. Eine Einführung, Berlin: Querverlag 2005, S. 187.

Vgl. Heinz-Jürgen Voß/Salih A. Wolter: Queer und (Anti-)Kapitalismus, Stuttgart: Schmetterling Verlag 2013. 
werden, weil die jährlich stattfindenden CSD-Paraden bis heute eine zentrale Rolle für schwul-lesbische, aber auch queere Politiken spielen. ${ }^{4}$

Tatsächlich kommt der Darstellung von »Stonewall« als Teil queerer Bewegungsgeschichte in zweifacher Hinsicht eine besondere Bedeutung zu. Erstens handelt es sich dabei um mehr als nur eine bewegungsgeschichtliche Situierung des im Zentrum der Arbeit stehenden queeren Aktivismus. Denn - und hier wird vorgegriffen - die Strukturanalyse des Text- und Bildmaterials der fünf im Zentrum der Arbeit stehenden queeren Projekte hat gezeigt, dass die Erinnerungsveranstaltungen zu den »Stonewall Inn«-Protesten (die CSD-Paraden) das dort am meisten diskutierte Thema sind. Deshalb ist die im Folgenden ausgeführte Darstellung der »Stonewall Inn«-Proteste gleichzeitig Teil der bewegungsgeschichtlichen Situierung und diskursiver Kontext der später folgenden Analyse. Zweitens unterscheidet sich die Form, in der dieser (Nicht-)Teil einer queeren Bewegungsgeschichte erzählt wird, von den darauffolgenden Abschnitten. Sie verweist exemplarisch auf die gleichzeitige Unmöglichkeit und Notwendigkeit, eine queere Geschichte zu schreiben.

Die folgende Darstellung der »Stonewall Inn«-Proteste ist eine Rekonstruktion verschiedener wissenschaftlicher und aktivistischer Erzählungen, die jeweils um »Hegemonie« ringen. Foucault zufolge kann das Verhältnis von Diskurs und Wirklichkeit »weder in Begriffen der Widerspiegelung noch in den Begriffen der Ideologie, noch in den Begriffen der Rationalisierung erklärt und analysiert werden «. ${ }^{5}$ Vielmehr geht es darum $\mathrm{zu}$ fragen, weshalb eine bestimmte Wirklichkeit von einem bestimmten Wahrheitsspiel begleitet wird und »welche Funktionen und Effekte« dieses »Regime der Veridiktion« jeweils hat. ${ }^{6}$ Anstatt die »wahre« Geschichte der Proteste in der »Christopher Street« zu skizzieren, wird im Folgenden, im Versuch, der von Foucault genannten Problematik Rechnung zu tragen, die Geschichte der »Stonewall Inn«-Proteste entlang zweier umkämpfter Narrative erzählt. Diese Form kann für die darauffolgenden Darstellungen queerer Bewegungsgeschichte - von der »Gay Liberation« über die Aids-Krise in den USA bis hin zu einer Bewegungsgeschichte im deutschsprachigen Raum - jedoch nicht aufrechterhalten werden. Das liegt zum einen daran, dass es kein mit »Stonewall«vergleichbares diskursives Ereignis gibt, und zum anderen daran, dass die queere Bewegungsgeschichte jenseits von »Stonewall« vorwiegend der bewegungsgeschichtlichen Situierung dient und nicht auch gleichzeitig zentraler diskursiver Kontext der später folgenden Analyse ist.

\subsubsection{Stonewall 1969: Umkämpfte Erinnerungspolitiken}

Die verschiedenen Erzählungen $\mathrm{zu}$ »Stonewall« verschwimmen, überschneiden und durchkreuzen sich. Es ist daher nicht möglich eine eindeutige Einordnung der

\footnotetext{
$4 \quad$ In Kapitel sieben wird ausführlich dargestellt, wie die CSD-Paraden im aktuellen deutschsprachigen queeren Aktivismus verhandelt werden.

5 Michel Foucault: Subjektivität und Wahrheit. Vorlesung am Collège de France 1980-1981, Berlin: Suhrkamp Verlag 2016, S. 318.

6 Ebd., S. $318 f$.
} 
unterschiedlichen Erzählungen als »queer« oder nicht »queer«, hegemonial oder gegenhegemonial vorzunehmen. Gemeinsam ist ihnen, dass sie noch lange nicht Teil eines gesamtgesellschaftlichen Wissens sind. Die Proteste in der »Christopher Street « sind Teil einer marginalisierten Geschichte. Doch auch innerhalb dieser marginalisierten Geschichte gibt es bestimmte Erzählungen, die diese Geschichtsschreibung bestimmen. Es gibt eine bestimmte Version, "Stonewall« zu erinnern, die in der Community und auch darüber hinaus hegemonial geworden ist. Es handelt sich um eine Version, die wiederum Gegenerzählungen hervorgerufen hat. Beide Erzählungen - die hegemonial gewordene Geschichte und die Gegenerzählungen - verweisen konstitutiv aufeinander. Queere Gegengeschichten hätten wenig zu erzählen, wenn es nicht andere, zumeist einfachere (homogenere) Erzählungen gegeben hätte, die sich in die Geschichte eingeschrieben haben.

Im Folgenden sollen zwei besonders umkämpfte Narrative um die »Stonewall Inn«-Proteste aufgegriffen werden. Einerseits wird »Stonewall« zum Teil bis heute als Geburtsstunde des schwul-lesbischen Aktivismus bezeichnet. Andererseits wurden die »Stonewall Inn«-Proteste zumeist als Kämpfe von Homosexuellen in die Geschichte eingeschrieben. Anhand dieser beiden Narrative - »Stonewall« als Geburtsstunde und "Stonewall« als schwul-lesbischer Kampf - sollen die Aushandlungen, die um die Erinnerung an das Ereignis »Stonewall« stattfinden, skizziert werden. Der Fokus wird dabei vor allem auf den Gegenerzählungen liegen, ohne den Anspruch, diese umfassend oder chronologisch nachzuzeichnen.

\section{Das Geburtsstundennarrativ}

In vielen Erzählungen gelten die »Stonewall Inn«-Proteste im August 1969 als die Geburtsstunde des schwul-lesbischen Aktivismus. In der Nacht zum 27. Juni hat es - wie so oft - eine Polizeirazzia in einer (von der Mafia betriebenen) Bar für Homosexuelle und Trans*-Personen in New York - dem »Stonewall Inn« in der »Christopher Street « - gegeben. Die Besucher*innen der Bar und die umstehenden Menschen ließen sich in dieser Nacht die Gewalt durch die Polizei nicht länger gefallen und lieferten sich tagelange Kämpfe mit der Polizei. Im Anschluss an diese Proteste formierte sich die „Gay Liberation «-Bewegung. ${ }^{7}$ Seither gelten die Kämpfe in der Christopher Street als die Geburtsstunde des schwul-lesbischen Aktivismus. Der Mythos von »Stonewall« als "starting point« des homosexuellen Aktivismus hat sich bereits während der Kämpfe in die Geschichte eingeschrieben. Craig Rodwell, ein homophiler Aktivist, der zufällig an der Bar vorbeilief, als die Kämpfe begonnen hatten, produzierte für den nächsten Tag Flyer, die "Stonewall« als das »emblematische Ereignis ${ }^{8}$ festschreiben, von dem sie (die Flyer) sprachen: »The nights of Friday, June 27, 1969 and Saturday, June 281969 will go down in history as the first time that thousands of Homosexual men and women

7 Vgl. John D'Emilio: Sexual Politics, Sexual Communities. The Making of a Homosexual Minority in the United States, 1940-1970, Chicago: University of Chicago Press 1998.

8 Scott Bravmann: »Queere Fiktionen von Stonewall«, in: Andreas Kraß (Hg.), Queer denken. Gegen die Ordnung der Sexualität, Frankfurt a.M.: Suhrkamp Verlag 2005, S. 240-274, hier S. 240. 
went out into the streets to protest the intolerable situation which has existed in New York City for many years. «9

Queere Gegenerzählungen hinterfragen allerdings, dass mit »Stonewall« alles begonnen habe. Sie verweisen unter anderem darauf, dass es chronologisch gesehen bereits vor "Stonewall« spontane, länger andauernde Kämpfe gegen Polizeigewalt gegeben habe. Das Geburtsstundennarrativ, so die implizite wie explizite Kritik solcher Gegenerzählungen, markiere einen Anfang, der kein Anfang war, weil es schon vorher Kämpfe, aber auch (schwul-lesbische) Strukturen gegeben habe, die in einer Ursprungserzählung unsichtbar bleiben. Ein Geburtsstundennarrativ lässt außen vor, dass es bereits vor den Protesten in der Christopher Street Bewegungen gegeben hat, die die Möglichkeitsbedingungen sowohl für die Proteste selbst als auch dafür, dass diese sich (auf eine bestimmte Art und Weise) in die Geschichte einschreiben konnten, geschaffen haben. Entgegen einer solchen Geburtsstundenerzählung verortet zum Beispiel die Historikerin Susan Stryker den Beginn des homosexuellen politischen Aktivismus in San Francisco in den 1950er-Jahren. Zu dieser Zeit waren die zwei führenden homophilen Initiativen $»$ Mattachine Society ${ }^{10}$ und $»$ Daughters of Bilitis $\aleph^{11}$ in San Francisco beheimatet und in den 1960ern wurden in San Francisco weitere sogenannte homophile Organisationen gegründet. Zudem gab es dort in dieser Zeit bereits eine ausgeprägte Drag-Kultur, als deren bekanntester Repräsentant José Sarria gilt. ${ }^{12}$

Die "Stonewall Inn«-Proteste als Geburtsstunde des schwul-lesbischen Aktivismus zu erinnern, markiert einen Bruch mit den zuvor bestehenden homophilen Bewegungen. ${ }^{13}$ Die homophilen Organisationen galten - so Annamarie Jagose - größtenteils als eher konservativ. Im Gegensatz zu der später folgenden Homobefreiungsbewegung standen sie für assimilatorische Politiken. So waren beispielsweise viele der homophilen Aktivist*innen nicht geoutet oder es wurde die Diagnose Homosexualität als Krankheit akzeptiert. Insgesamt ging es den homophilen Aktivist*innen vor allem darum, Homosexualität in der Gesellschaft akzeptierbarer zu machen. Diese assimilatorischen Politiken ließen sich kaum mit den Zielen der später folgenden »Homobefreiungsbewegungen « vereinbaren, in denen das stolze nach außen Tragen einer schwul-lesbischen Identität im Mittelpunkt stand. ${ }^{14}$ Indem die Geburtsstundenerzählung mit der Vergangenheit bricht, gelingt es, eine kohärente, stolze schwul-lesbische politische Identität herzustellen. Der Historiker John D'Emilio hält dem entgegen, dass die homophilen Bewegungen Strukturen geschaffen hätten, die »Stonewall« überhaupt erst ermöglichten: »The work of homophile activists mattered; they set the stage for Stonewall and

9 Elizabeth A. Armstrong/Suzanna M. Crage: »Movements and Memory. The Making of the Stonewall Myth«, in: American Sociological Review 71 (2016), S. 724-751, hier S. 738.

10 Die»Mattachine Society « war eine Männer-Organisation, die 1950 in Los Angeles gegründet wurde und zwischen 1954-1957 nach San Francisco umzog.

11 Die »Daughters of Bilitis« sind eine lesbische Organisation, die 1955 von Del Martin und Phyllis Lyon gegründet wurden.

12 Susan Stryker: San Francisco 2004, https://www.glbtqarchive.com/ssh/san_francisco_S.pdf vom 11.10.2019.

13 A. Jagose: Queer Theory, S. 47.

14 Ebd., S. $43 f$. 
gay liberation. ${ }^{15}$ Insbesondere die Öffentlichkeit und der Grad der Vernetzung der homophilen Bewegung haben es möglich gemacht, »Stonewall« in die kollektiven Erinnerungspolitiken einzuschreiben. Eine wichtige Rolle spielte dabei die $1967 \mathrm{im}$ Kontext des homophilen Aktivismus entstandene nationale Zeitschrift »The Advocate«. Sie hat maßgeblich dazu beigetragen, den 27. Juni in New York in die Geschichte einzuschreiben. Die landesweite Vernetzung in und zwischen den homophilen Bewegungen hat zudem eine zentrale Rolle bei der Organisation der ersten Erinnerungsparaden zu den "Stonewall Inn«-Protesten gespielt. ${ }^{16}$ Eine Erzählung, die »Stonewall« als Geburtsstunde des schwul-lesbischen Aktivismus markiert, vergisst, dass diese Strukturen notwendig waren. Unsichtbar bleibt dabei nicht nur, dass die »Gay Liberation« aus nicht immer ganz so stolzen assimilatorischen Strukturen hervorgetreten ist, die Teile ihrer Geschichte und damit Teil von dem, was sie geworden ist - sind. Sondern auch, dass es der im homophilen Aktivismus vorhandenen sozialen und ökonomischen Ressourcen bedurfte, um »Stonewall« in die Geschichte einzuschreiben. Dieser Aspekt wird dann relevant, wenn verhandelt wird, warum die Kämpfe in der Christopher Street und nicht andere Proteste in die Geschichte eingeschrieben wurden. Es ist aber auch von Gewicht in Bezug auf das Wir, das in einer bestimmten Art und Weise des Einschreibens von »Stonewall« in die Geschichte hergestellt wird.

Ähnlich wie im Fall der homophilen Bewegungen lässt ein Geburtsstundennarrativ auch die zahlreichen Verknüpfungen zu den anderen Bewegungen der 1960er-Jahre außen vor. Diese - insbesondere die Bürgerrechtsbewegung - hatten einen wichtigen Einfluss auf die "Stonewall Inn«-Proteste. Viele "Stonewall«-Aktivist*innen hatten sich in den damaligen Bewegungen radikalisiert ${ }^{17}$ und Strategien und Slogans, insbesondere aus der »Black Power«-Bewegung, wurden von den Protestierenden in der Christopher Street angeeignet. Die »Gay Liberation« hat zudem später stark von der politischen Infrastruktur, wie beispielsweise den regelmäßig stattfindenden Protestveranstaltungen, der anderen Bewegungen profitiert. ${ }^{18}$ Andere Kämpfe und Bewegungen, die schon vor den »Stonewall Inn«-Protesten bestanden, hatten diese Kämpfe überhaupt erst möglich gemacht. Eine Geburtsstundenerzählung markiert einen »starting point«, der all diese der »Gay Liberation« vorausgehenden Bewegungen außen vor lässt. Neben der Tatsache, dass eine Geburtsstundenerzählung einen Bruch markiert, der Kontinuitäten zu anderen Bewegungen unsichtbar macht, ignoriert diese Art, »Stonewall« zu erinnern, auch, dass es chronologisch gesehen schon vor dem Juni 1969 Aufstände gegen Polizeigewalt gegeben hat.

In San Francisco kam es bereits 1966 in »Compton's Cafeteria« zu spontanen, länger andauernden Aufständen gegen die willkürliche Polizeigewalt. ${ }^{19}{ }{ }_{\text {Compton's Cafe- }}$

15 J. D'Emilio: Sexual Politics, Sexual Communities, S. 258.

16 E. A. Armstrong/S. M. Crage: Movements and Memory, S. 738.

17 Leslie Feinberg: Interview: Sylvia Rivera 1998, https://www.queerbible.com/queerbible/2017/10/8/i nterview-sylvia-rivera-by-leslie-feinberg vom 11.10.2019.

18 J. D’Emilio: Sexual Politics, Sexual Communities, S. $224 \mathrm{f}$

19 In Los Angeles hatten sich 1966/67 und 1968 Proteste gegen die gewaltvollen Polizeirazzien in den Bars »Black Cat« und »The Patch« organisiert. Darüber hinaus gab es in Los Angeles auch Versuche, im Anschluss an ein Hass-Verbrechen an einem homosexuellen Mann, der im März 1969 von einem Polizeibeamten an einem Parkplatz totgeschlagen wurde, Erinnerungsveranstaltungen zu 
teria« war ein 24-Stunden Coffeeshop, der bei »queens«, "gay hustlers«, »street kids« und "hair fairies« sehr beliebt war. ${ }^{20}$ Ein Aktivist* erzählt, dass eine Dragqueen, die gerade von einem Polizisten gepackt worden sei, diesem Kaffee ins Gesicht geschüttet habe, woraufhin sie von den anderen "gays « in der Bar in ihrem Widerstand unterstützt worden sei. Auch am Morgen streikten die Menschen gemeinsam, weil »Compton's Cafeteria« beschlossen hatte, die Dragkings und -queens nicht mehr hereinzulassen. In San Francisco gab es also chronologisch betrachtet sogar noch vor »Stonewall « affektiv aufgeladene, spontane und gewaltvolle Kämpfe, geführt von verschiedenen Gruppen, die sich gegen die routinierte Polizeigewalt und Schikane wehrten. Allerdings wurde der Aufstand von "Compton's Cafeteria« weder in den homophilen Medien noch in den Mainstream-Medien erwähnt. Die mit medialen Ressourcen ausgestatteten homophilen Aktivist*innen hatten Angst, dass die Sexarbeiter*innen und Dragqueens ihre eigenen Forderungen und Politiken unterminieren würden. ${ }^{21}$ Die Tatsache, dass der Aufstand medial überhaupt nicht beachtet wurde, zeigt, dass die marginalisierteren Gruppen, die an diesen Protesten maßgeblich beteiligt waren, nicht die Möglichkeiten hatten, sich Gehör zu verschaffen. Sie konnten, mit Spivak gesprochen, nicht sprechen, weil sie von jeglichen Linien sozialer Mobilität abgeschnitten waren. ${ }^{22}$ Es ist - wieder mit Spivaks ${ }^{23}$ Worten - der umfangreichen Recherche einer Intellektuellen zu verdanken, die die Verantwortung dafür übernommen hat, die Geschichte der Aufstände in San Francisco zu rekonstruieren, sodass sie nicht ganz vergessen wurde: »Strykers archaeological efforts recovered this event for the historical record. $\ll^{24}$

Die Gegenerzählungen zu einer Erinnerung von »Stonewall« als Beginn des schwullesbischen Aktivismus verweisen darauf, dass mit »Stonewall« nicht »alles « angefangen hat. Allerdings markieren die damaligen Proteste in der Christopher Street den Beginn der »Gay Liberation«-Bewegung. Indem eine Geburtsstundenerzählung mit den der "Geburt« vorausgehenden Bewegungen und Kämpfen bricht, kann sie ein homogenes Subjekt der »Gay Liberation« konstituieren. Es wird ein politisches Subjekt hergestellt, das von Anfang an stolz war und nichts mit den »queens«, "gay hustlers«, »street kids« und »hair fairies« von »Compton's Cafeteria« gemeinsam hat. Letzteres äußert sich auch in dem gängigen Narrativ von »Stonewall« als schwul-lesbischer Kampf.

\section{Das schwul-lesbische Narrativ}

Die Flyer des homophilen Aktivisten Craig Rodwell sprechen nicht nur von »Stonewall« als erstem Protest gegen die Polizeigewalt, sondern sie schreiben auch fest, wer damals

organisieren. Armstrong und Crage vermuten, dass die massive repressive Polizeigewalt in Los Angeles maßgeblich dazu beitrug, dass die Proteste nicht die gleichen Auswirkungen und die gleiche Reichweite wie »Stonewall« hatten. Gleichzeitig waren die Aktivist"innen in Kalifornien der Bewegung zuliebe bereit, trotz der in ihren Augen falschen Ursprungserzählung, die Erinnerungsparaden auch an ihren Orten mitzutragen. E. A. Armstrong/S. M. Crage: Movements and Memory, S. 740 .

20 Ebd., S. 732.

21 Ebd., S. 733.

22 G. C. Spivak: Can the Subaltern Speak?

23 G. C. Spivak: Geschichte.

24 E. A. Armstrong/S. M. Crage: Movements and Memory, S. 733. 
auf die Straße gegangen ist: "The nights of Friday, June 27, 1969 and Saturday, June 281969 will go down in history as the first time that thousands of Homosexual men and women went out into the streets to protest the intolerable situation which has existed in New York City for many years. ${ }^{25}$ Ähnlich wurde auch in der zeitnahen Berichterstattung der Zeitschrift »The Advocate« von aufständischen »homosexuals, gays und boys « geschrieben. ${ }^{26}$ Die »Stonewall Inn«-Proteste gelten als homosexuelle - mehr schwule als lesbische - Kämpfe. Dieses Narrativ hält sich zu großen Teilen bis heute. ${ }^{27}$ Queere Gegenerzählungen hinterfragen das homogene homosexuelle Wir, das in dieser Art, "Stonewall $\mathrm{zu}$ erinnern, hergestellt wird. Allerdings sind diese alternativen Erzählungen selbst heterogen: Zunächst einmal gibt es viele Versuche, die maßgebliche Beteiligung von Trans*-Personen in den Mittelpunkt der Erzählungen zu stellen. Andere kritisieren vor allem, dass die ethnischen Zugehörigkeiten unsichtbar bleiben. ${ }^{28}$ Lesbische Frauen fragen wiederum, ob sie nicht fälschlicherweise in eine Geschichte männlich dominierter Kämpfe und deren Repräsentationspolitiken eingeschlossen wurden. ${ }^{29}$ Im Folgenden werden vor allem solche Gegenerzählungen in den Blick genommen, die den Einschluss von Trans*-Personen und People of Color in die »Stonewall Inn«-Erzählungen einfordern.

Seit den 1990er-Jahren kämpfen vor allem Trans*-Aktivist"innen und Aktivist*innen of Color darum, die Rolle von Sylvia Rivera und Marsha P. Johnson ${ }^{30}$ bei den Protesten und der sich im Anschluss an »Stonewall« formierenden »Gay Liberation« sichtbar zu machen. Vor allem Sylvia Riveras Zeitzeug*innenberichte haben dazu beigetragen, eine Geschichte von schwulen Kämpfen zu verkomplizieren. In einem Interview mit Leslie Feinberg erzählt Rivera von den Kämpfen und wer daran beteiligt war: »It was street gay people from the Village out front - homeless people who lived in the park in Sheridan Square outside the bar - and then drag queens behind them and everybody behind us. «11 Im Gegensatz zu einer homosexuellen Erzählung wird hier also die maßgebliche Beteiligung von Drags, Trans*-Personen, Sexarbeiter*innen, wohnungslosen Jugendlichen und People of Color hervorgehoben. Die Zeitzeug*innenberichte der lesbischen afroamerikanischen Aktivist*in Irene Monroe betonen vor allem die Rolle der Schwarzen Community bei den Protesten. In ihrem Artikel »Dis-remembering

Ebd., S. 738.

Jessi Gan: »Still at the Back of the Bus«. Sylvia Rivera's Struggle«, in: Centro Journal XIX (2007), S. 124-139, hier S. 132.

Vgl. Tanja Vogler: »Pride-Paraden. Queere Erinnerungspolitiken«, in: Verena Sperk/Sandra Altenberger/Katharina Lux et al. (Hg.), Geschlecht und Geschlechterverhältnisse bewegen. Queer/Feminismen zwischen Widerstand, Subversion und Solidarität 2020, S. 73-98.

Bravmann spricht von einer Ignoranz in den 70ern und einer Sichtbarmachung in den 8oern. S. Bravmann: Queere Fiktionen von Stonewall, S. 248.

Ebd., S. 252.

Sylvia Rivera und Marsha P. Johnson stehen sowohl für die fehlende Repräsentation von Trans*Personen und Dragqueens als auch für die fehlende Repräsentation von People of Color. Sie waren zudem beide Sexarbeiter*innen, oft wohnungslos sowie drogen- und alkoholabhängig und repräsentieren somit auch viele andere Gruppen, die bei den Protesten beteiligt waren.

31 L. Feinberg: Interview: Sylvia Rivera. 
Stonewall« berichtet sie, wie sie selbst als damals Siebzehnjährige gemeinsam mit ihrer ganzen Nachbarschaft aus Harlem aufbrach, um die Protestierenden in Greenwich Village zu unterstützen. ${ }^{32}$ Die Erfahrungen der Schwarzen Community in den 7oern waren zum einen von den Erfolgen der Bürgerrechtsbewegung, zum anderen von repressiver Polizeigewalt geprägt. Während erstere, wie bereits erwähnt, eine der zentralen Möglichkeitsbedingungen der »Stonewall«-Proteste waren, ermöglichte letztere Allianzen über Differenzen hinweg: „During this tumultuous decade of black rage and white police raids, knee-jerk responses to each other slights easily to the stage for a conflagration, creating both instantaneous and momentary fighting alliances in these black-communities - across gangs, class, age, ethnicity and sexual orientation against police brutality. ${ }^{33}$

Scott Bravmann betont, dass solche Gegenerzählungen auch zu einem anderen über die Frage, wer dabei war, hinausgehenden - Verständnis der damaligen Proteste führen: »Genau weil Ethnizität einen Unterschied macht [...], haben die ethnischen Dynamiken einen großen Einfluss darauf, wie wir die Ausschreitungen verstehen. « ${ }^{34}$ Mit Blick auf die Zeitzeug*innenerzählungen von Rivera und Monroe kann in Anlehnung an Judith Butler davon gesprochen werden, dass Prekarität - verstanden als ungleiche Verteilung von Gefährdetheit - Allianzen möglich gemacht hatte. ${ }^{35}$ Damals waren es zumeist nicht die schwulen weißen Männer, sondern die Butches, Dragqueens, Trans* Menschen und Sexarbeiter*innen, viele von ihnen of Color, die besonders von Polizeigewalt betroffen waren: ${ }^{36}$ »We always felt that the police were the real enemy. We expected nothing better than to be treated like we were animals - and we were. We were stuck in bullpen like a bunch of freaks. We were disrespected. A lot of us were beaten up and raped. $\ll^{37}$ An den Protesten waren maßgeblich Gruppen beteiligt, für die die Proteste auch eine Frage von Leben und Tod waren. Marsha P. Johnson überlebte diesen Kampf nicht. Sie wurde 1992 nach einer CSD-Parade tot im Hudson River aufgefunden. ${ }^{38}$ Es waren - solchen Gegenerzählungen zufolge - zum größten Teil besonders gefährdete Gruppen, die im Zuge der »Stonewall Inn«-Proteste auf die Straße gegangen sind. Indem sie gemeinsam die Verletzlichkeit ihrer Körper offenlegten, haben sie ihr Recht auf Unversehrtheit geltend gemacht. Aber auch die affektive Dimension der Proteste wird unterschätzt, wenn diejenigen, in deren Körper sich die rassistische, homophobe und trans*phobe Polizeigewalt primär einschrieben hat, nicht mitgedacht werden. Die Razzien im Sommer 1969 waren keine einmaligen Razzien, sondern Teil einer ständigen Routine. Ohne dass es geplant oder organisiert wurde, war es dieser Tag (der 27. Juni), an dem die Dragqueens Schnapsgläser auf die Polizist*innen geworfen haben, an dem

Irene Monroe: »Dis-membering Stonewall«, in: The Huffington Post vom 26.06.2013, https://bit.ly /3lesymQ vom 11.10.2019.

Ebd.

S. Bravmann: Queere Fiktionen von Stonewall, S. 242.

]. Butler: Anmerkungen zu einer performativen Theorie der Versammlung, S. 183.

Das hing auch mit der damaligen Cesetzeslage zusammen, nach der es verboten war, gegengeschlechtliche Kleidung zu tragen. J. Gan: >Still at the Back of the Bus<, S. 131.

L. Feinberg: Interview: Sylvia Rivera.

Michael Bronski: »Sylvia Rivera: 1951-2002«, in: Z Magazine vom 04.2002, https://zcomm.org/zma gazine/sylvia-rivera-1951-2002-by-michael-bronski/ vom 11.10.2019. 
der schwule puerto-ricanische Mann die Butch beschützte, die verhaftet werden sollte, an dem die Dragqueen die Polizist*innen mit ihren High Heels zusammenschlug und Sylvia Rivera den zweiten Molotowcocktail warf. ${ }^{39}$ D'Emilio warnt davor, über eine Erzählung der Wut die politischen und sozialen Kontexte außen vor zu lassen, die die Möglichkeitsbedingungen für den Aufstand herstellten. Die Wut war aber - folgen wir den Erzählungen von Monroe und Rivera -, genau wie die politischen und sozialen Kontexte, ebenfalls von Gewicht. Erzählungen, die ein schwul-lesbisches Narrativ "queeren«, heben die Prekarität und affektive Dimension der damaligen Kämpfe hervor. Einer solchen Leseart zufolge waren die »Stonewall Inn«-Proteste insofern bereits "queer«, als es Kämpfe waren, in denen Allianzen auf Basis einer "gemeinsam geteilten Verletzlichkeit « und Wut geknüpft wurden. Körper sind gemeinsam auf der Straße erschienen, um unbetrauerbare Verluste zu politisieren und gemeinsam das Recht auf Unversehrtheit geltend zu machen. Eine Geschichte des Ereignisses »Stonewall« als schwul-lesbischer Kampf schließt bestimmte Gruppen aus der Darstellung aus und verschließt damit auch bestimmte Dimensionen dieser Kämpfe.

»Stonewall« als Geburtsstunde und als schwul-lesbischen Kampf zu erinnern, bedeutet, ein homogenes, kohärentes Wir an den Beginn der schwul-lesbischen Befreiungsbewegungen zu stellen. Dass dieses Wir nicht ganz so kohärent und auch nicht ausschließlich - vielleicht nicht einmal maßgeblich - homosexuell war, heben die verschiedenen Gegenerzählungen zu diesen Narrativen hervor. Sie verweisen auch auf eine Lesart, nach der die »Stonewall Inn«-Proteste keine schwul-lesbischen Identitätspolitiken, sondern affektive Kämpfe unter den Bedingungen der Prekarität waren, die folglich im Sinne Judith Butlers auch als "queer« bezeichnet werden können. Auch in der »Gay Liberation«-Bewegung, die sich im Anschluss an »Stonewall« formierte, schreibt sich fort, was mit dem »Stonewall«-Mythos festgeschrieben wurde.

\subsubsection{Gay Liberation: Bündnisse - Spaltungen - Identitätspolitiken}

Im Anschluss an die »Stonewall Inn«-Proteste formierte sich zeitnah in New York die »Gay Liberation Front (GLF)«. Sie gilt als eine der ersten Gruppen der homosexuellen Befreiungsbewegung. Die »GLF« wurde auf einer im Anschluss an die »Stonewall Inn«Proteste organisierten Versammlung der homophilen Organisation »Mattachine Society« gegründet. Die Gruppe hatte sich von ebendieser abgespalten. Auslöser war ein Streit um die Solidarisierung mit inhaftierten »Black Panther«-Mitgliedern, die viele homophile Aktivist*innen nicht mittragen wollten. ${ }^{40}$ Im Gegensatz zum homophilen Aktivismus suchte die »GLF « von Anfang an Verbündete in anderen Bewegungen ${ }^{41}$ und

39 J. Gan: sStill at the Back of the Bus«.; J. D'Emilio: Sexual Politics, Sexual Communities; Martin B. Duberman: Stonewall, New York: Penguin Books 1994; Michael Bronski: A Queer History of the United States, Boston: Beacon Press 2011.

40 M. B. Duberman: Stonewall, S. 217.

41 Paula-Irene Villa: »Von >sex perverts` zu >Liberation NOW tät innerhalb der neuen sozialen Bewegungen (50er bis 70er)«, in: Paula-Irene Villa/Lutz Hieber (Hg.), Images von Gewicht. Soziale Bewegungen, Queer Theory und Kunst in den USA, Bielefeld: transcript Verlag 2007, S. 81-123, hier S. 108f. 
die gay is good-Politiken der »Mattachine Society« wurden von out and proud-Politiken abgelöst. Das Coming-out - das stolze nach außen Tragen der eigenen Identität - wurde zu einem zentralen Moment des politischen Protests der »Gay Liberation «. ${ }^{42}$ Das »Gay Manifesto«, das 1970 von der »Red Butterfly Cell ${ }^{43}$ der »Gay Liberation Front« veröffentlicht wurde, verweist exemplarisch darauf, dass es innerhalb der »GLF« bereits politische Visionen gab, die »queere « Züge aufwiesen. ${ }^{44}$ Es werden nicht nur Mehrfachdiskriminierung und hegemoniale Männlichkeiten reflektiert, sondern auch Bündnisse gefordert: »But not every straight is our enemy. Many of us have mixed identities, and have ties with other liberation movements: women, blacks, other minority groups [...]. And face it: we can't change Amerika alone. « ${ }^{45}$ Doch die im Manifest geäußerte Haltung entsprach - so zeigten spätere Konflikte - nicht unbedingt der »Wirklichkeit«. Die anfangs sehr breite Allianz der »Gay Liberation Front« bröckelte schnell und die »GLF« spaltete sich in immer differenzierter agierende kleine Gruppen.

Bereits zwei Jahre nach der Gründung löste sich die »GLF« wieder auf. Zum einen gab es Konflikte um die Frage, mit wem und zu welchem Preis Bündnisse geschlossen werden sollten. So war beispielsweise die homophobe Rhetorik der »Black Panthers « vielen ein Dorn im Auge. ${ }^{46}$ Andere kritisierten die Unterstützung des sozialistischen Projekts Kuba - das bekannt war für homophobe Politiken - durch ihre Bündnispartner*innen. ${ }^{47}$ Zum anderen spaltete sich die »GLF auch aufgrund interner Grenzziehungsprozesse: Einerseits führte die Marginalisierung von Aktivist"innen of Color und Trans*-Personen in der »GLF« dazu, dass diese schon relativ früh eigene Gruppen gründeten. So formierte sich bereits 1970 die Gruppe »The Third World Gay Revolution «, ebenso wie die Organisation "STAR (Street Trans Activist Revolutionaries)«, die sich für die Unterstützung von wohnungslosen Trans*-Jugendlichen einsetzte. ${ }^{48}$ Andererseits entstand mit den »Gay Activist Alliances (GAA) « eine Gruppe, die moderatere Menschen zusammenführte. ${ }^{49}$ Lesbische Frauen schlossen sich aufgrund der maskulinen Rhetorik und der männlichen Dominanz in der »Gay Liberation« zumeist eher der Frauenbewegung an. ${ }^{50}$ Doch auch die Frauenbewegung hatte ihre Schwierigkeiten mit lesbischen Frauen. Entsprechend veröffentlichten die »Radical Lesbians« auf der zweiten nationalen Frauenkonferenz 1970 als Antwort auf die Ausschlüsse der Frauenbewegung - sie waren zuvor als »Lila Bedrohung« bezeichnet worden ${ }^{51}$ - das bis heute

42 A. Jagose: Queer Theory, S. 47.

43 »The Redbutterfly Cell« war eine marxistische Zelle innerhalb der »GLF«.

44 Wittman benutzt in seinem Manifest zudem bereits den Begriff »queer « als individuelle Selbstbezeichnung.

45 Carl Wittman: A Gay Manifesto 1970, http://library.gayhomeland.org/ooo6/EN/A_Gay_Manifesto. htmvom 15.09.2020, S. 7.

46 Terence Kissack: »Freaking Fag Revolutionaries: New York's Gay Liberation Front, 1969-1971«, in: Radical History Review 62 (1995), S. 104-134, hier S. 116.

Ebd., S. 126.

48 M. B. Duberman: Stonewall, S. 235.

49 M. Bronski: A Queer History of the United States, S. 211.

50 T. Kissack: Freaking Fag Revolutionaries: New York's Gay Liberation Front, 1969-1971, S. 112.

51 "Lavender Menance« bezieht sich auf eine Aussage von Betty Friedman, mit der diese die lesbischen Frauen als eine Bedrohung für die Frauenbewegung markierte. 
bekannte Manifest "Women Identified Women.$^{52}$ In dem Manifest forderten sie ein politisches Subjekt Frau, das sich nicht in Bezug auf den Mann konstituiert: »And yet in popular thinking, there is really only one essential difference between a lesbian and other women: that of sexual orientation - which is to say, when you strip off all the packing, you must finally realize that the essence of being a >woman< is to get fucked by $\mathrm{men} \ll .53$

Es kam also schon früh zu Spaltungen und Fragmentierungen einer homosexuellen Befreiungsbewegung, die es sich anfangs zum Ziel gemacht hatte, in breiten Allianzen zu arbeiten. Die gesamte »Gay Liberation« verlor im Laufe der 1970er-Jahre an Radikalität. Die Fragmentierung in kleinere Gruppen führte sich sowohl innerhalb der Homobefreiungsbewegungen als auch innerhalb der lesbisch-feministischen Bewegungen fort. $\mathrm{Ab}$ Mitte der 7oer-Jahre spricht Jagose von einem Ende schwul-lesbischer Politiken, in denen gemeinsam das sex-gender-System angeprangert wird. Stattdessen formierten sich einzelne kleine Gruppen, die auf lokale Schauplätze fokussiert immer »identitätspolitischer a agierten. Statt radikaler Befreiung traten vermehrt die Herstellung einer einheitlichen Homosexuellen-Identität und die Forderungen nach Anerkennung dieser Gruppen in den Vordergrund. Es ging nicht mehr so sehr darum, die Verhältnisse radikal auf den Kopf zu stellen, sondern ein Stück vom Kuchen abzubekommen. Gegen Ende der 7oer-Jahre war die lesbisch-schwule Subkultur weit verbreitet und erlebte zunehmende Toleranz. ${ }^{54}$

In der homosexuellen Befreiungsbewegung, die an die »Stonewall Inn«-Proteste anschloss, wurden bereits Anfang der 1970er-Jahre sowohl im schwul dominierten politischen Kontext der »Gay Liberation« als auch im lesbischen Feminismus - ebenso wie im Schwarzen Feminismus ${ }^{55}$ - Räume des Denkens eröffnet, auf die die Formierung queerer Politiken in den 8oer-Jahren zurückgreifen konnten. Es zeigt sich aber auch, wie Versuche, Bündnisse zu schließen, an identitätspolitischen Ausschlüssen scheiterten.

\subsubsection{Aids-Aktivismus: Queer Time}

Die Möglichkeitsbedingungen von »queer werden an der Schnittstelle von politischen Dynamiken, Theorieproduktion und politischen, ökonomischen sowie kulturellen Veränderungen in den USA verortet. Sabine Hark bezeichnet dieses Zusammenkommen von Möglichkeitsbedingungen als "Queer Time«: »Der Moment der Artikulation von queer. ${ }^{56}$ In bewegungspolitischen Kontexten stellte die Kritik an den Ausschlüssen sexueller Identitäten eine wichtige Möglichkeitsbedingung dar. ${ }^{57}$ Auf der Ebene der Theorieproduktion schufen Artikel von Autor*innen wie Adrienne Rich und Monique

P.-I. Villa: Von >sex perverts zu >Liberation NOW $₫$ ! Positionen zu Ceschlecht und Sexualität innerhalb der neuen sozialen Bewegungen (50er bis 70er), S. 104.

53 Radicalesbians: The Woman-Identified Woman 1970, https://www.historyisaweapon.com/defcon1 /radicalesbianswoman.htmlvom 11.10.2019.

55 Vgl. hierzu Kapitel eins.

56 S. Hark: Queer Studies, S. 291.

Diese Debatten werden auch als feministische »sex wars« bezeichnet. 
Wittig die theoretischen Grundlagen zur Formierung queerer Politiken, indem sie Sexualität als Kategorie in den Vordergrund rückten. ${ }^{58}$ Aber auch Michel Foucaults erster Band von »Sexualität und Wahrheit«, der 1976 erschienen ist, spielt in Bezug auf die »Möglichkeitsbedingungen der Artikulation von queer « eine bedeutende Rolle. ${ }^{59} \mathrm{Er}$ zeigt auf, wie das Dispositiv der Sexualität Subjekte reguliert, und kritisiert die einfache Bejahung von ebendieser als Möglichkeit der Befreiung. ${ }^{60}$ Diese Debatten führten zu einer Verschiebung des politischen Diskurses hin zur Dimension Sexualität als eigene sozial hergestellte Kategorie, die einen zentralen Aspekt späteren queeren Denkens ausmacht. ${ }^{61}$ Auf gesellschaftspolitischer Ebene formierte sich im Kampf gegen die Sozialpolitik Kennedys, aber auch gegen die Errungenschaften der Frauen-, Friedens- und Bürgerrechtsbewegung Ende der 70er-Jahre eine neue Rechte. Mit der Wahl von Reagan 1980, der politisch unter anderem für militärische Aufrüstung und Steuersenkung stand, manifestierte sich ein konservativer Backlash. ${ }^{62}$

In diese Zeit fällt auch der Beginn der sogenannten Aids-Krise. 1981 wurde der erste Aids-Fall beschrieben und 1983 konnte der Virus nachgewiesen werden. Die Tatsache, dass diese Krankheit bei vielen Schwulen ausbrach, führte dazu, dass sie zunächst als Gay Related Disease bezeichnet wurde. Die neue Rechte nutzte in Koalition mit der Kirche das Aufkommen von Aids, um die Krankheit und den Tod symbolisch mit Homosexualität zu verknüpfen. Das Virus, so die rechte Rhetorik, galt als Bestrafung für eine Gesellschaft, die etwas Sittenloses wie Homosexualität toleriert. Politisch wurde die Gesundheitskrise von Reagan jahrelang ignoriert beziehungsweise genutzt, um schwule Bars, Sexclubs und Badehäuser zu schließen oder Sexarbeiter*innen zu verhaften. ${ }^{63}$ Infolge dieser Ignoranz gab es bereits 19831.476 Aids-Tote in den USA. 1987 stieg die Zahl dann auf 11.932 an und 1990 waren es 31.129 Aids-Tote. ${ }^{64}$ Als Antwort auf den Backlash und den Tod formierte sich der Aids-Aktivismus, der auch als Beginn des queeren Aktivismus gilt.

\section{»Act Up«-Aktivismus}

$\mathrm{Zu}$ Beginn der Aids-Krise entstanden zunächst einmal aus der LGBT-Community heraus Organisationen zur Unterstützung von Menschen mit Aids jenseits ihrer sexuellen Orientierung. Eine solche Organisation war beispielsweise die "Gay Men's Health Crisis (GMHC)« in New York oder die »People With AIDS Coalition (PWA)«. Diese Einrichtungen führten Beratungen, Gesundheitsversorgung sowie Hausbesuche durch und leisteten Bildungs- und Aufklärungsarbeit. Anfangs gab es allerdings auch innerhalb

Paula-Irene Villa: »Kritik der Identität, Kritik der Normalisierung - Positionen von Queer Theory«, in: Paula-Irene Villa/Lutz Hieber (Hg.), Images von Gewicht. Soziale Bewegungen, Queer Theory und Kunst in den USA, Bielefeld: transcript Verlag 2007, S. 165-190, hier S. 168.

59 S. Hark: Queer Studies, S. 298.

60 Vgl. M. Foucault: Der Wille zum Wissen.

61 P.-I. Villa: Kritik der Identität, Kritik der Normalisierung - Positionen von Queer Theory, S. 175.

62 Lutz Hieber: »Politisierung der Queer Culture durch ACT UP«, in: Paula-Irene Villa/Lutz Hieber (Hg.), Images von Cewicht. Soziale Bewegungen, Queer Theory und Kunst in den USA, Bielefeld: transcript Verlag 2007, S. 191-235, hier S. 192.

64 M. Bronski: A Queer History of the United States, S. 224. 
der Community einige Versuche, Sexualität zu regulieren. Die frühen HIV-PräventionsGuidelines warnten vor gefährlichen sexuellen Aktivitäten wie S/M-Praktiken und forderten weniger häufige Sexualpartner*innenwechsel. Safer-Sex-Kampagnen gaben diesen Kämpfen um die Regulierung von Sexualität eine andere Richtung. Beginnend mit dem 1983 verfassten Pamphlet »How to Have Sex in an Epidemic: One Approach « von Richard Berkowitz und Michael Callen änderte sich die zuvor eingeschlagene Richtung. ${ }^{65}$ Die folgenden Safer-Sex-Kampagnen trugen dazu bei, die Übertragung von HIV einzudämmen, und setzten gleichzeitig der Regulierung und Repression von Sexualität in und außerhalb der Community etwas entgegen. ${ }^{66}$ Während die meisten LGBTOrganisationen Gesundheitseinrichtungen waren, die noch vornehmlich auf rechtlicher Ebene Kämpfe führten, entstand mit »Act Up (Aids Coalition to Unleash Power)« 1987 die erste »direct action group« gegen Aids. Zu dieser Zeit waren bereits viele Menschen unzufrieden mit den eher unpolitischen Gesundheitseinrichtungen.

»Act $U p$ « formierte sich als breite Koalition von verschiedenen Menschen, die unterschiedlich von der Aids-Krise betroffen waren. Die vorher separat agierenden Gruppen von Lesben, Schwulen, Trans*-Menschen, People of Color und anderen erkannten, dass in der Konfrontation mit einem tödlichen Virus Identität keine Rolle spielte: »these waves of dying friends - trumped the long history of divisions within the LGBT community. « ${ }^{67}$ Menschen aus dem Gesundheitswesen, Familien und Freund"innen sowie andere von Aids Betroffene schlossen sich »Act Up« an. Maxine Wolfe - eine »Act Up«Aktivist"in - berichtet, dass "Act Up«, entgegen häufiger Beschreibungen als weiß und schwul, vielfältig gewesen sei. Die einzige Gruppe, die nicht bei »Act Up« zu finden gewesen sei, waren ihr zufolge heterosexuelle Männer. ${ }^{68}$ Douglas Crimp - ebenfalls "Act Up«-Aktivist" - erzählt in einem Interview, dass »Act Up« nicht nur linke, politisch radikalisierte Menschen zusammenbrachte, sondern auch Menschen umfasste, die keinen linken politischen Hintergrund hatten: »It was basically a bunch of queers, and some of them had traditional leftist backgrounds - especially the women - but a lot of them didn't. Many members had rather mainstream politics, but they had AIDS and they were fighting for their lives. ${ }^{69}$

Die Politiken von »Act Up« waren aber nicht nur insofern »queer«, als Identität nicht länger das Band war, das die Gruppe zusammenhielt, sondern auch insofern, als sich der Ort des Politischen verschob. Während sich vorher Homosexualität in den Subkulturen und im Privaten abgespielt hatte, wurden beginnend mit »Act Up« Homosexualität und deren unbetrauerbaren Verluste öffentlich politisiert. »Act Up«-Politiken zielten darauf $a b$, die Verknüpfung zwischen Aids und Homosexualität zu entkoppeln und ein alternatives beziehungsweise überhaupt ein Wissen zu Aids herzustellen. Gleichzei-

Jennifer Brier: »Aids and Action (1980-1990s) «, in: Don Romesburg (Hg.), The Routledge History of Queer America, New York: Routledge 2018, S. 95-106, hier S. 97.

66 M. Bronski: A Queer History of the United States, S. $228 f$.

67 Ebd., S. 228.

68 Maxine Wolfe/Laraine Sommella: This Is about People Dying: The Tactics of Early ACT UP and Lesbian Avengers in New York City, Queers in Space: Communities, Public Places, Sites of Resistance 1997, https://actupny.org/documents/earlytactics.htmlvom 15.09.2020. 
tig forderten sie vehement eine Gesundheitspolitik, die die Krankheit ernst nimmt. ${ }^{70}$ Mit ihren vielfältigen politischen Aktionen und der medialen Aufmerksamkeit war »Act Up«, wie Michael Bronski festhält, eine der bis dahin effektivsten aktivistischen Gruppen, die aus der schwul-lesbischen Community hervorgegangen waren. ${ }^{71}$ Die politischen Strategien von »Act Up« zielten vor allem darauf $a b$, eine breite Öffentlichkeit zu erreichen. Das Künstler*innenkollektiv»Gan Fury« spielte dabei eine zentrale Rolle. Eines der bekannteren Beispiele von politischen Interventionen der Gruppe ist das von ihnen entwickelte »Silence=Death «-Plakat, dessen Logo später zum Markenzeichen des "Act Up«-Aktivismus wurde. Auf schwarzem Hintergrund war ein pinkes Dreieck und darunter in weißer fetter Schrift "Silence=Death « zu sehen. Das pinke Dreieck symbolisierte den auf den Kopf gestellten rosa Winkel aus der Nazizeit. ${ }^{72}$ Unter dem "Silence=Death «-Slogan stand folgender Text: »Warum schweigt Reagan zu Aids? Was geht tatsächlich im Center for Disease Control, der Federal Drug Administration und im Vatikan vor? Schwule und Lesben sind nicht entbehrlich ... Benutze deine Macht ...Wähle ... Boykottiere ...Verteidige dich ... Wende Wut, Angst und Kummer in Aktivität.« In und durch die kreative Aneignung von Werbestrategien und die Verknüpfung von Kunst und Grafikdesign gelang es »Gan Fury« und später auch anderen Kollektiven, in den öffentlichen Diskurs zu intervenieren. Es folgten in den nächsten Jahren viele ähnliche Kampagnen inner- und außerhalb des »Act Up«-Aktivismus. ${ }^{73}$

»Act Up« setzte sich zudem früh dafür ein, auch frauenspezifische Themen, die im Kontext der Aids-Krise mehrfach unsichtbar waren, in die öffentlichen Diskurse einzuspeisen. An der Schnittstelle von race und gender stellte die Kriminalisierung von HIV einen weiteren wichtigen Ort des politischen Kampfes dar. Bereits 1988 wurden »HIVspecific criminalisation laws« etabliert, die die Übertragung von HIV als Verbrechen deklarierten. Der »Ryan White Care Act« sorgte zwei Jahre später dafür, dass die einzelnen Bundestaaten HIV kriminalisieren mussten, wollten sie finanzielle Unterstützung für HIV/Aids-Care und Bildung. ${ }^{74}$ Ein bekanntes und auch von »Act Up« politisiertes Beispiel für die Verknüpfung von HIV und Kriminalität ist der Fall von Greg Smith, einem Schwarzen, schwulen HIV-positiven Mann. Greg Smith wurde, wie Cindy Patton ausführlich berichtet, wegen versuchten Mordes zu 25 Jahren Haft verurteilt, weil er angeblich einen Polizisten gebissen hatte. Dass HIV nicht durch Beißen übertragen werden kann, spielte in diesem Urteil keine Rolle. »Act Up Philadelphia« organisierte Proteste und Foren, um Smith zu unterstützen. Smith starb noch im Gefängnis. ${ }^{75}$ Obwohl »Act Up« auch rassistische Praktiken der Kriminalisierung politi-

70 L. Hieber: Politisierung der Queer Culture durch ACT UP, S. 196

71 M. Bronski: A Queer History of the United States, S. 233.

72 Für die mit der Vereinnahmung von Symbolen aus der Nazizeit einhergehende Verharmlosung ebendieser wurde das Logo auch kritisiert.

73 L. Hieber: Politisierung der Queer Culture durch ACT UP, S. $194 f$.

74 Che Gossett: »We Will Not Rest in Peace: AIDS Activism, Black Radicalism, Queer and/or Trans Resistance«, in: Jinthana Haritaworn/Adi Kuntsman/Silvia Posocco (Hg.), Queer Necropolitics, London: Routledge 2014, S. 31-48, hier S. 34.

75 Cindy Patton: „Concealed Weapon. PWA Greg Smith Is Serving 25 Years for Attempted Murder. His Crime? Allegedly Biting a Corrections Officer«, in: POZ - Health, Life and HIV vom 11.1998, https:// www.poz.com/article/Concealed-Weapon-1657-8008vom 11.10.2019. 
sierte, ${ }^{76}$ blieben andere Aspekte an der Schnittstelle race und gender in den damaligen queeren Aids-Politiken unsichtbar. Die Verteilung der 1987 veröffentlichten Krankheitsfälle verweisen auf eine verhältnismäßig höhere Zahl von Aids-Kranken in den Blackund Hispanic-Communitys. ${ }^{77}$ Queere Aids-Politiken, so die Kritik, basierten jedoch zumeist auf anglostereotypen Vorstellungen von Homosexualität und Bisexualität, die an den Lebensrealitäten vieler Menschen aus den Hispanic-Communitys vorbeigingen und diese entsprechend auch nicht erreichen konnten. ${ }^{78}$

\section{"Queer Nation"}

Während »Act Up«-Politiken sich vornehmlich auf Aids bezogen, ist mit »Queer Nation $(Q N)$ « 1990 eine Gruppe aus »Act Up« hervorgegangen, die den öffentlichen sexuellen Diskurs generell adressierte. ${ }^{79}$ "Queer Nation « gilt zudem als erste politische Gruppe, die sich das ehemalige Schimpfwort ${ }^{80}$ "queer « als Selbstbezeichnung angeeignet hat. ${ }^{81}$ In ihrem Manifest »The Queer Nation Manifesto« beschreibt »Queer Nation« die Aneignung von »queer« als einen affektiven subversiven Akt:

»Using >queer is a way of reminding us how we are perceived by the rest of the world. It's a way of telling ourselves we don't have to be witty and charming people who keep our lives discreet and marginalized in the straight world. We use queer as gay men loving lesbians and lesbians loving being queer. Queer, unlike gay, doesn't mean male. And when spoken to other gays and lesbians it's a way of suggesting we close ranks, and forget (temporarily) our individual difference because we face a more insidious common enemy. Yeah, queer can be a rough word but it is also a sly ironic weapon we can steal from the homophobe's hands and use against him. $\ll^{82}$

Das Manifest wurde 1990 erstmals auf der Pride-Parade in New York ausgeteilt. Bereits in diesem Manifest wird »queer« als ein Begriff festgeschrieben, der Bündnisse jenseits von Identitäten möglich macht. Die Interventionen von » $\mathrm{QN}$ « zielten vor allem darauf ab, Homosexualität in den öffentlichen Raum zu tragen und so die Bedeutung amerika-

Im Kontext der Kriminalisierung von HIV setzte sich »Act Up« ebenfalls für die im HIV-Camp in Guantanamo eingesperrten Haitianer*innen ein, die aufgrund ihrer Krankheit nicht in den USA Asyl beantragen durften, aber wegen bestehendem internationalem Asylrecht auch nicht zurückgeschickt werden konnten. C. Cossett: We Will Not Rest in Peace: AIDS Activism, Black Radicalism, Queer and/or Trans Resistance, S. 37. Halperin/Henry Abelove/Michele A. Barale (Hg.), The Lesbian and Gay Studies Reader, New York: Routledge 1993.

78 Ebd., S. 121.

79 Lauren Berlant/Elizabeth Freeman: »Queer Nationality«, in: boundary 219 (1992), S. 149, hier S. 155.

80 A. Jagose: Queer Theory, S. 97.

81 Bereits 1910 und 1939 bezeichneten sich Männer, die Interesse an anderen Männern hatten, als queer Cloria Anzaldúa hat schon vor »Queer Nation«, bereits 1987, den Begriff »queer«verwendet. A. Jagose: Queer Theory, S. 97. 
nischer Nationalität umzuschreiben: ${ }^{83}$ Strategien wie »Kiss-ins «, "Queer Nights Out « ${ }^{84}$ oder die »Pink Panther Patrol ${ }^{85}$ wurden genutzt, um den amerikanischen heterosexuellen Raum, der nur solange bestehen bleibt, wie Queers schweigen und unsichtbar sind, aufzubrechen. »Queer Nation« konnte allerdings nicht - so Lauren Berlant und Elizabeth Freemans Kritik - die glamouröse Homogenität des amerikanischen Traums überwinden. Berlant und Freeman fassen die Politiken von » $\mathrm{QN}$ « folgendermaßen zusammen:

»[T] he production of a queer counterpublic of traditional national icons, the official and useful spaces of everyday life, the ritual places of typical public pleasure (parades, malls, bars, and bodies), and the collective identities consumers buy in the mode of mass culture. [...] QN shows that the boundaries that might secure distinctions between sexual populations are local (like neighbourhoods), normative (like taxonomies), and elastic (like latex). $\aleph^{86}$

Ähnlich wie »Act Up« und entgegen den Forderungen in ihrem Manifest, wird auch "Queer Nation« dafür kritisiert, ökonomische und antirassistische Aspekte vernachlässigt $\mathrm{zu}$ haben. ${ }^{87}$ Trans*-Personen, vor allem Trans*-PoC und Trans*Sexarbeiter"innen, waren besonders hart von der Aids-Krise betroffen. Während es in den 8oern mit Ausnahme der FTM-Community, ${ }^{88}$ wenig Transgender-Aktivismus gab, boten Allianzen, wie sie die Aids-Krise erforderte, neue Möglichkeiten für den Trans*-Aktivismus. ${ }^{89} 1992$ veröffentlichte Leslie Feinberg ihr einflussreiches Pamphlet »Transgender Liberation: A Movement Whose Time Has Come«, und ebenfalls in diesem Jahr wurde »Transgender Nation (TN)« ins Leben gerufen. »Transgender Nation« ist aus "Queer Nation San Francisco« hervorgegangen. Die Gruppe versuchte, Trans*Themen mit den anderen Themen aus der queeren Community zusammenzubringen, um eine inklusive politische Agenda zu vertreten. ${ }^{90}$

Im Zuge der Aids-Krise hat sich ein politischer Aktivismus formiert, der im Kampf gegen den Tod Allianzen jenseits eindeutiger Identitäts-Kategorien begründete. Im Zentrum dieser neuen Politiken stand die Regulierung und Normalisierung von Sexualität. Dabei ging es immer auch darum, die Grenze zwischen Öffentlichem und Privatem zu unterlaufen. Im deutschsprachigen Raum hatte die Aids-Krise andere

83 L. Berlant/E. Freeman: Queer Nationality, S. 156.

84 »Queer Nation« eignete sich temporär »straight bars« an, um die heterosexuelle Normativität zu irritieren.

85 Mit Walkie-Talkies und einem schwarzen T-Shirt mit pinkem Dreieck ausgestattet, liefen die »Pink Panthers« an verschiedenen öffentlichen Orten Patrouille. Durch diese Aktionen konnte »QN« gleichzeitig einen sicheren öffentlichen Ort einsetzen und fordern.

86 L. Berlant/E. Freeman: Queer Nationality, S. 170.

87 Ebd., S. 171.

88 »TM«steht für »female to male«.

89 Susan Stryker: Transgender Activism 2004, https://www.glbtqarchive.com/ssh/transgender_activis m_S.pdf vom 15.09.2020.

90 Ein*e Interaktivist*in beschreibt, wie er*sie durch »Transgender Nation « wiederum motiviert worden sei, eine Inter"-Organisation aufzubauen. Chase, Cheryl: »Hermaphrodites with Attitude: Mapping the Emergence of Intersex Political Activism«, in: Susan Stryker/Stephen Whittle (Hg.), The Transgender Studies Reader, New York: Routledge 2006, S. 316-330. 
Auswirkungen. Entsprechend hat sich der queere Aktivismus dort nicht eigenständig als Antwort auf Aids formiert, sondern ist aus den USA übernommen worden.

\subsection{Die Entwicklung queerer Politiken im deutschsprachigen Raum}

Queerer Aktivismus im deutschsprachigen Raum geht zum einen auf einen Prozess der Aneignung theoretischer wie politischer Perspektiven aus den USA zurück, zum anderen auf eine jeweils eigene Geschichte sexualpolitischer und geschlechterpolitischer Bewegungen. Nachdem die Aids-Krise und der politische Backlash sowie eine bereits länger bestehende identitätspolitische Subkultur die Entstehung von queeren Politiken in den USA ermöglichten, werden Bezug nehmend auf die USA mögliche Unterschiede herausgearbeitet, die darauf verweisen, warum sich im deutschsprachigen Raum womöglich queere Politiken nicht derart formierten. Das bedeutet vor allem, die Auswirkungen der Aids-Krise und den Umgang der jeweiligen politischen Bewegungen mit Aids in den Blick zu nehmen, um zumindest teilweise nachvollziehen zu können, warum "queer « sich im deutschsprachigen Raum nicht aus der gleichen Notwendigkeit heraus artikuliert hat wie in den USA. Eine bewegungsgeschichtliche Situierung von queeren Politiken im deutschsprachigen Raum soll aber nicht ausschließlich in Bezug zu einer US-amerikanischen Geschichte geschrieben werden. Es soll auch eine, den jeweiligen Ländern eigene, queere Bewegungsgeschichte skizziert werden, die den im Zentrum der vorliegenden Arbeit stehenden aktuellen queeren Projekten ebendort vorausgegangen ist.

In der folgenden Darstellung geht es vor allem darum, die Formierung und Institutionalisierung sowie die wichtigsten Themen und Konfliktlinien schwul-lesbischer und anderer Gruppen und Bewegungen, die aktuellen queeren Aktivismen im deutschsprachigen Raum vorausgehen, ohne Anspruch auf absolute Vollständigkeit nachzuzeichnen. Wie bereits in der Einleitung erwähnt wurde, stehen fünf queere Projekte - die Milchjugend, das Jugendnetzwerk Lambda BB, LesMigraS, TransInterQueer e.V. und die Türkis Rosa Lila Villa im Zentrum der später folgenden Untersuchung. Die bewegungsgeschichtliche Kontextualisierung dieser fünf für die empirische Untersuchung ausgewählten Einrichtungen endet zumeist in den 1990er-Jahren. Das ist der Moment, ab dem queer als eine Politikform im deutschsprachigen Raum angeeignet wurde. Es ist aber auch der Moment, in dem sich drei der im Zentrum der Arbeit stehenden Projekte - LesMigraS, die Rosa-Lila-Villa und das Jugendnetzwerk Lambda BB - formierten. Im Fall der Schweiz organisierten sich queere Politiken später und langsamer, und auch das im Zentrum der Analyse stehende Projekt - die Milchjugend - ist erst im Jahr 2012 gegründet worden. Entsprechend gehen hier die Ausführungen über die 1990er-Jahre hinaus. Zudem werden, ebenfalls über die 1990er-Jahre hinausgehend, die Anfänge des Trans*Aktivismus - in dessen Kontext das fünfte im Zentrum der vorliegenden Arbeit stehende Projekt, TransInterQueer e.V., verortet werden kann - sowie des migrantischen und Schwarzen sexualpolitischen Aktivismus herausgearbeitet. Diesen Formen des queeren Aktivismus kommt in der bewegungsgeschichtlichen Situierung jedoch vergleichsweise wenig Raum zu, da diese Geschichte jung, marginalisiert und entsprechend wissenschaftlich weniger umfangreich aufgearbeitet ist. Die Entwicklungsgeschichte queerer 
Politiken ist zudem in den drei Ländern - Deutschland, Österreich und Schweiz - unterschiedlich umfangreich ausgearbeitet. Die Schwulen- und Lesbenbewegung ist in Deutschland bereits in vielen wissenschaftlichen Arbeiten auf unterschiedlichen Ebenen und aus verschiedenen Perspektiven rekonstruiert und eingeordnet worden. ${ }^{91}$ Für die Schweiz konnten im Vergleich weit weniger wissenschaftliche Aufarbeitungen sexualpolitischer Bewegungen gefunden werden. ${ }^{92}$ Zumeist handelt es sich nur um kurze, überblicksartige Beiträge oder um auf geographische, zeitliche oder auf spezifische Gruppen beschränkte Analysen. Hier musste vermehrt auf Quellen direkt aus der Bewegung zurückgegriffen werden, wie beispielsweise Bewegungszeitschriften (z.B. »Lesbenfront «) oder zumeist von ehemaligen Aktivist*innen erstellte Internetportale (z.B. »Das Wiki zur Lesbengeschichte der Schweiz«). ${ }^{93}$ Für Österreich, speziell für Wien, hat Ulrike Repnik ${ }^{94}$ eine sehr ausführliche Geschichte der Lesben- und Schwulenbewegung vorgelegt, die als primäre Quelle verwendet wurde. ${ }^{95}$ Die unterschiedlich umfangreiche wissenschaftliche Aufarbeitung spiegelt sich auch in den folgenden Darstellungen wider.

91 Vgl. Gabriele Dennert/Christiane Leidinger/Franziska Rauchut (Hg.): In Bewegung bleiben. 100 Jahre Politik, Kultur und Ceschichte von Lesben, Berlin: Querverlag 2007; Christiane Leidinger: »Frühe Debatten um Rassismus und Antisemitismus in der (Frauen- und) Lesbenbewegung in den $1980 e r$ Jahren der BRD«, in: Beiträge zu einer Ceschichte der pluralen Linken. Theorien und Bewegungen nach 1968 (2010), S. 24-29; Patrick Henze: Schwule Emanzipation und ihre Konflikte. Zur westdeutschen Schwulenbewegung der 1970er Jahre, Berlin: Querverlag 2019; S. Haunss: Identität in Bewegung.

92 Zu nennen wären hier beispielsweise: Ruth Ammann: Politische Identitäten im Wandel. Lesbischfeministisch bewegte Frauen in Bern 1975 bis 1993, Nordhausen: Traugott Bautz 2009; Christina Caprez/Yv E. Nay: »Frauenfreundschaften und lesbische Beziehungen. Zur Geschichte frauenliebender Frauen in Graubünden«, in: Silke Redolfi/Silvia Hofmann/Ursula Jecklin (Hg.), fremdeFrau: Frauen- und Geschlechtergeschichte Graubünden, Zürich: Neue Zürcher Zeitung Libro 2008; Madeleine Marti: »Lesbische Lichtblicke. Die erste Lesbenorganisation und die erste lesbisch-schwule Zeitschrift in der Schweiz der $30 e r$ Jahre«, in: Eva Rieger/Hiltrud Schroeder (Hg.), >Diese Frau ist der Rede wert،. Festschrift für Luise Pusch, Herbolzheim: Centaurus-Verlag 2004, S. 86-98; Beat Gerber: Lila ist die Farbe des Regenbogens, Schwestern, die Farbe der Befreiung ist rot: die homosexuellen Arbeitsgruppen in der Schweiz (HACH) von 1974-1995. Zugl.: Bern, Univ., Lizentiatsarbeit, 1997, Köniz: Ed. Soziothek 1998.

93 Das Wiki zur Lesbengeschichte der Schweiz, https://I-wiki.ch/L-World_-_Das_Wiki_zur_Lesbenge schichte_der_Schweiz. Ich möchte mich bei allen Personen, die mich bei der Literatursuche zur Schweizer Bewegungsgeschichte über den Mail-Verteiler »Sündikat « unterstützt haben (YvE. Nay, Alecs Recher, Schelley Berlowitz), sowie Corinne Rufli bedanken.

94 Ulrike Repnik: Die Geschichte der Lesben- und Schwulenbewegung in Österreich, Wien: MilenaVerlag 2006.

95 Nachdem Repnik sich in ihrem Werk hauptsächlich auf Wien bezieht und auch die Türkis Rosa Lila Villa in Wien angesiedelt ist, bleiben die Ausführungen zu Österreich hauptsächlich auf Wien beschränkt. 


\subsection{1 Österreich}

In Deutschland, Österreich und der Schweiz hatten sich bereits Ende des 18. Jahrhunderts ausgehend von Magnus Hirschfeld ${ }^{96}$ erste schwul-lesbische Zusammenschlüsse gegründet. ${ }^{97}$ Das Berlin der 1920er-Jahre galt generell als eine Metropole der schwullesbischen und Trans*-Community, in der sich Schwule, Lesben und »Transvestiten" viele subkulturelle Räume angeeignet hatten. ${ }^{98}$ Während die Schweiz zu Zeiten des Nationalsozialismus der letzte verbliebene Fluchtpunkt homosexuellen Lebens und homosexueller Öffentlichkeit war, sind in Deutschland und Österreich nicht nur die politischen (kollektiven) Strukturen, sondern fast das komplette homosexuelle und Trans* Leben ausgelöscht worden. ${ }^{99}$

Im Vergleich zu anderen »westlichen« Ländern entwickelte sich in Österreich der homosexuelle Aktivismus relativ spät. Die Lesben- und Schwulenbewegung formierte sich dort erst Ende der 1970er-, Anfang der 1980er-Jahre. Also zu einer Zeit, in der die USA auf eine lange Geschichte von homophiler Bewegung über »Gay Liberation« bis hin $\mathrm{zu}$ schwul-lesbischen Identitätspolitiken zurückblickte. Die späte Neu-Organisation der durch den Nationalsozialismus zerstörten homosexuellen Strukturen kann, Repnik zufolge, auch auf die repressive Gesetzeslage in Österreich zurückgeführt werden. In Österreich war Homosexualität bis 1971 verboten. Repnik vermutet auch, dass der große Einfluss der Katholischen Kirche bis in die 1970er-Jahre dazu beigetragen hat, dass die Schwulen- und Lesbenbewegung in Österreich vergleichsweise spät entstand. ${ }^{100}$ Erst in der Kreisky-Ära - eine Zeit, die generell produktiv für die Mobilisierung sozialer Bewegungen in Österreich war - wurde im Zuge der kleinen Strafrechtsreform das generelle Homosexualitätsverbot aufgehoben und durch vier neue Paragraphen ersetzt. ${ }^{101}$ Die Aufhebung des Verbots der Homosexualität, Vorbilder aus dem Ausland, die Gründung europäischer schwul-lesbischer Organisationen wie beispielsweise »ILGA (International Lesbian, Gay, Bisexual, Trans and Intersex Association)« und die Formierung anderer sozialer Bewegungen ermöglichten es, ab Ende der 7oer-Jahre in Österreich erste schwul-lesbische Strukturen zu schaffen. ${ }^{102}$

Bereits 1897 gründete Magnus Hirschfeld das »Wissenschaftlich humanitäre Komitee (WHK) «, das sich auf Grundlage alternativer wissenschaftlicher Erkenntnisse gegen die Kriminalisierung von Homosexualität einsetzte.

U. Repnik: Die Geschichte der Lesben- und Schwulenbewegung in Österreich, S. $130 f$.

Karl-Heinz Steinle: »Räume für Träume. Auf der Suche nach Treffpunkten und anderen Freiräumen für Lesben, Schwule und Trans*personen in der frühen Bundesrepublik«, in: Carolin Küppers/Martin Schneider (Hg.), Orte der Begegnung - Orte des Widerstands. Zur Geschichte homosexueller, trans*geschlechtlicher und queerer Räume, Hamburg: Männerschwarm Verlag 2018, S. 45-64, hier S. $45 f$.

99 U. Repnik: Die Geschichte der Lesben- und Schwulenbewegung in Österreich, S. 75

100 Ebd., S. 83f.

101 Zum einen war männliche Prostitution und die gleichgeschlechtliche Unzucht mit Minderjährigen verboten, zum anderen - das betrifft den Aufbau homosexueller Strukturen - war es verboten, Homosexualität zu bewerben oder Verbindungen zu gründen, die Homosexualität begünstigten. Ebd., S. $59 f$. 
Im Unterschied zu den USA ist »queer« ist in Österreich nicht als Antwort auf eine politische Notwendigkeit im Kampf um den Tod entstanden. Vielmehr ist dieser Begriff in den 1990er-Jahren nach Österreich übergeschwappt und wurde im Kontext des politischen Aktivismus oft übernommen, um eine inklusivere Form der Politik zu bezeichnen. ${ }^{103}$ Die Türkis Rosa Lila Villa, die bereits in den 1980er-Jahren aus den radikaleren Flügeln der homosexuellen Bewegung hervorgegangen ist, gilt heute neben anderen Gruppen, die sich mittlerweile in Österreich formiert haben, ${ }^{104}$ immer noch als wichtiger Raum des queeren Aktivismus.

\section{Die Schwulenbewegung}

Die erste homosexuelle Gruppe »Coming Out« formierte sich 1975. Sie löste sich aber einige Jahre später wieder auf, nachdem Konflikte, die Radikalität der Gruppe und die Frage nach der Inklusion von Frauen betreffend, unüberwindbar wurden. ${ }^{105}$ Es gelang dann 1980 einigen schwulen Männern mit der »Hosi« in Wien, trotz des bestehenden Vereinsverbots, den ersten rechtlich legalen Verein für homosexuelle Menschen zu gründen. Zentrale politische Ziele bestanden in den Forderungen nach rechtlicher Gleichstellung. Die »Hosi« fuhr zu Beginn noch eine zweigleisige Politik zwischen Anpassung und Gay-Pride, die sich in kleineren radikalen Aktionen äußerte. ${ }^{106}$ Diese Zweigleisigkeit löste sich dann, so Repnik, in Richtung angepasster bürgerlicher Politik auf. $^{107}$ Der 1982 gegründete »Rosa Wirbel« galt im Gegensatz zur »Hosi« als »radikale« schwule Gruppe, deren Aktionen an die »Act Up«-Politiken erinnern. Ihr Ziel war es, zu provozieren und mediale Aufmerksamkeit zu erreichen: »Dieser Haufen hatte schon zum Neujahrskonzert 1982 für einen >Rosa Wirbek gesorgt, und unvergessen sind die beiden Nackten (Florian Sommer und Robert Herz nur mit einer Fliege bekleidet), die pünktlich zur Polka >Die Emanzipierte im traditionsreichen Wiener Musikvereinssaal die Bühne stürmten und >Freiheit für Schwule ‘ forderten. " ${ }^{108}$ Die Aktionen des »Rosa Wirbel« gelten auch als Vorläufer der im selben Jahr aus der Hausbesetzer*innenbewegung entstandenen Rosa Lila Villa - eines der im Zentrum der vorliegenden empirischen Untersuchung stehenden Projekte. Die Rosa Lila Villa ${ }^{109}$ galt als erste Gruppe, in der schwul-lesbische Zusammenarbeit möglich war. ${ }^{110}$

103 C. M. Klapeer: queer.contexts, S. $109 \mathrm{ff}$.

104 Z.B. MiGay, Frauencafe*, Planet1o in Wien.

105 Ebd., S. 90.

106 So wurden auf der einen Seite ernste Cespräche mit politischen Vertreter*innen geführt, auf der anderen Seite wurden beispielsweise ab 1981 Erinnerungsveranstaltungen an die »Stonewall Inn «Proteste organisiert. Ebd., S. $156 \mathrm{f}$.

107 Ebd., S. 111.

108 Marty Huber: 25 Jahre andersrum. Die Rosa Lila Villa an der Linken Wienzeile 102. IC Kultur Wien 2007, https://igkultur.at/artikel/25-jahre-andersrum-die-rosa-lila-villa-der-linken-wienzeile-1 02 vom 24.06.2020.

109 Das Türkis ist erst später dazugekommen. Vgl. Kapitel vier.

110 U. Repnik: Die Geschichte der Lesben- und Schwulenbewegung in Österreich, S. 117. 


\section{Die Lesbenbewegung}

Lesbische Frauen in Österreich organisierten sich mit Ausnahme der Villa lange Zeit hauptsächlich im Kontext der Frauenbewegung. Im Rahmen von »AUF (Aktion unabhängiger Frauen)« entstand 1976 bereits die erste lesbische Gruppe. Zunächst gab es auch innerhalb der Frauenbewegung Konflikte zwischen lesbischen und heterosexuellen Frauen. ${ }^{111} \mathrm{Ab}$ den 8oer-Jahren wurden immer mehr lesbische Gruppen in der Frauenbewegung präsent und im Rahmen des "Frauen-Kultur Zentrums (FKZ)« entstand das erste Szenecafé, das der politischen Mobilisierung und dem Austausch diente. ${ }^{112}$ 1981 formierte sich auch eine lesbische Gruppe innerhalb der »Hosi«, was ebenfalls Konfliktpotential mit sich brachte. ${ }^{113}$ Schwul-lesbische Zusammenarbeit zeichnete sich in den 8oern/90ern dadurch aus, dass es neben dem gemeinsamen Ziel, die vier Paragraphen abzuschaffen, einige heftig umkämpfte Konfliktlinien gab: Die Dominanz schwuler Männer, die mangelnde Akzeptanz der Bedürfnisse von lesbischen Frauen - zum Beispiel nach geschlechtergerechter Sprache oder eigenen Räumen -, der Umgang mit Pädophilie und die Forderung nach der Ehe für alle stellten zentrale Streitpunkte dar. ${ }^{114}$

\section{Die Aids-Krise}

Die Aids-Krise kam in Österreich drei bis vier Jahre später als in den USA an. 1983 gab es den ersten Aids-Fall, also zu Zeiten, in denen sich schwul-lesbische Gruppen erst zu organisieren begannen. Allerdings hatte die Aids-Krise andere Auswirkungen als in den USA, was zum einen auf den politischen Kontext zurückzuführen ist, zum anderen aber auch darauf, dass die Aktivist"innen Wissen zu Präventionsarbeit aus den USA zur Verfügung hatten. Zudem gab es in Österreich im Gegensatz zu den USA ein Sozialversicherungssystem, das die Gesundheitsversorgung von vielen Menschen möglich machte. ${ }^{115}$ Auch die frühe Zusammenarbeit von homosexuellen Organisationen und dem Gesundheitsbereich trug ihren Teil dazu bei, dass die österreichische Politik anders auf Aids reagierte. Bereits 1983 brachte die "Hosi« in Wien in Zusammenarbeit mit Ärzt"innen die europaweit erste Informationsbroschüre zu Aids heraus. Die Medizin war überfordert mit einer Gesundheitskrise, auf die sie keine Antwort hatte, und homosexuelle Initiativen hatten Wissen zu dem Thema und Interesse am Aufbau von Strukturen gegen die Krankheit. So wurde bereits 1985 auf Initiative der »Hosi« die erste Aids-Hilfe in Österreich gegründet. Auch die österreichische Politik war froh, dass diese Gruppen die Aufgabe übernahmen, Strukturen aufzubauen, und unterstützte die Aids-Hilfen entsprechend. ${ }^{116}$ Das führte dazu, dass homosexuelle Gruppen von Anfang

111 Ebd., S. 93.

112 Ebd., S. 102.

113 Einige Männer in der »Hosi« waren der Meinung, die »Hosi« als einen rein schwulen Ort bewahren zu müssen, und aus der Frauenbewegung wurde von einigen die Zusammenarbeit mit den Männern kritisiert. Ebd., S. 137.

114 Ebd., S. 139-147.

115 Christine M. Klapeer: queer.contexts. Entstehung und Rezeption von Queer Theory in den USA und Österreich. Masterarbeit, Innsbruck 2003, S. $105 \mathrm{f}$.

116 Alois Unterkircher: »Die Gründung der Landesstelle Tirol der Österreichischen AIDS-Hilfe 1986. Ein Beispiel für die gelungene Zusammenarbeit zwischen Betroffenen, medizinischen Expertln- 
an Einfluss auf die Aids-Politiken hatten. So waren sie auch bei der Ausarbeitung des österreichischen Aids-Gesetzes 1986 beteiligt. Im Gegensatz zu den USA, in denen es zur Kriminalisierung von an Aids erkrankten Menschen kam, konnte im österreichischen Gesetzesentwurf die staatliche Verantwortung für die Gesundheitskrise festgeschrieben werden. ${ }^{117}$ Die homosexuellen Initiativen arbeiteten zudem von Beginn an gegen eine Verknüpfung von Homosexualität und Aids. ${ }^{118}$

Es entstanden neue Gruppen und Zeitschriften, wie der Verein »Safe Way« und die Zeitschrift »Xtra«. In Wien wurde sogar ein Ableger von »Act Up« - »Act Up Wien« - gegründet. Die Gruppe übernahm einige bekannte Kampagnen aus den USA, wie beispielsweise die »Bloody Hand«-Kampagne, die das Künstler*innen-Kollektiv »Gan Fury« entwickelt hatte. ${ }^{119}$ So verbreitete »Act Up Wien«, den »Bloody Hand«-Slogan auf die österreichischen Gegebenheiten umschreibend, Postkarten, auf denen stand: »Die ÖVP und die katholische Kirche haben Blut an den Händen. « ${ }^{120}$ Provokative, direkte Aktionen, wie die von »Act Up Wien« und dem »Rosa Wirbel«, sollten aber die schwullesbischen Politiken Ende der 1980er- und Anfang der 1990er-Jahre nicht bestimmen. Die Regulierung von Sexualität und die Forderungen nach Gleichheit vor dem Gesetz wurden dominant. Die Zusammenarbeit mit anderen Gruppen und Organisationen im Zuge der Aids-Krise förderte wiederum die Institutionalisierung der Bewegung. ${ }^{121}$ Entsprechend haben sich queere Politiken, wie sie in den USA mit »Act Up« und »Queer Nation « in Verbindung gebracht werden, so nicht in Österreich etabliert. Vielmehr setzten die damals bestehenden Gruppen auf Gleichstellungs- und Normalisierungspolitiken.

\section{Trans*- und migrantische Initiativen}

Bis Ende der 8oer-Jahre gab es in Wien, jenseits von vereinzelten subkulturellen Orten, wenig Räume für Trans*-Menschen und schon gar keine Anlauf- oder Beratungsstellen. Viele Trans*-Personen lebten in Österreich bis in die 1990er-Jahre zurückgezogen. Einige waren zumeist unsichtbar in der Friedensbewegung, der Frauenbewegung, der BDSM-Bewegung oder der schwul-lesbischen Bewegung aktiv. ${ }^{122}$ Die erste Trans* Gruppe in Österreich war die Gruppe »Transsexuellen Initiative Österreich «, die in den 1980ern in Graz entstanden ist und 1990 als Verein registriert wurde. Dem folgten Aufrufe für Vernetzungstreffen vor allem an hausbesetzten Orten in Wien wie beispielsweise der Rosa Lila Villa oder dem »WUK (Werkstättenkulturhaus)«. Bereits 1994 fand ein erstes Trans*-Filmfestival in Wien statt. Aus den informellen Treffen in der Villa sind

nen und Cesundheitspolitik?«, in: Bricolage: Innsbrucker Zeitschrift für Europäische Ethnologie 5 (2008), S. 141-164, hier S. 156.

117 Ebd., S. 154.

118 Ebd., S. 142.

119 L. Hieber: Politisierung der Queer Culture durch ACT UP, S. 198. Im Zuge dieser Kampagne wurde der rote Händeabdruck z.B. an Hauswänden zum Symbol für Kritik an der Politik und der Kirche, die für die Aids-Toten verantwortlich gemacht wurden.

120 U. Repnik: Die Geschichte der Lesben- und Schwulenbewegung in Österreich, S. 154.

121 Ebd., S. 155.

122 Persson P. Baumgartinger: Trans Studies. Historische, begriffliche und aktivistische Aspekte, Wien: Zaglossus Verlag 2017, S. 167. 
dann ab 1993 zwei Gruppen entstanden: der Verein »Transvestiten - Verein für Transvestiten und Transsexuelle ${ }^{123}$ und 1995 die Initiative »TransX«. Der Verein »TransX « nutzt bis heute die Räumlichkeiten der Villa und hat auch die Öffnung der Villa-Politiken für Trans*-Personen maßgeblich vorangebracht. ${ }^{124}$ Die Organisation von queeren migrantischen Personen und Queers of Color gibt es in Österreich erst seit den 2000erJahren. Mit der Gruppe »Vienna Mix« entstand 2004 eine der ersten selbstorganisierten migrantischen Gruppen in Wien, die sich zunächst ebenfalls in den Räumen der Villa traf. Auch in Linz organisierten sich bereits 2002 in der Gruppe »LesMAus (Lesbian Migrants in Austria)« lesbische Migrant"innen, die unter anderem Workshops zu Mehrfachdiskriminierung veranstalteten. ${ }^{125}$

\subsubsection{Deutschland}

Auch in Deutschland mussten schwul-lesbische Strukturen nach 1945 wiederaufgebaut werden, was allerdings etwas schneller als in Österreich von statten ging. Ähnlich wie in Österreich spielte auch dort die Liberalisierung rechtlicher Restriktionen - in dem Fall des Paragraphen $175^{126}$ - im Jahr 1969 eine wichtige Rolle für die Mobilisierung homosexueller Bewegungen. ${ }^{127}$ Die Aids-Krise hatte auch hier eine andere Auswirkung als in den USA und »queer « formierte sich in Deutschland nicht als Antwort auf die AidsKrise. »Queer« hat vor allem durch die akademisch-feministische Rezeption von Judith Butlers Werk »Gender Trouble« Einzug gehalten ${ }^{128}$, das insbesondere in (lesbisch-)feministischen Zusammenhängen diskutiert wurde. Insbesondere Sabine Hark ${ }^{129}$ brachte an der Schnittstelle von Wissenschaft und lesbischem Aktivismus schon früh queere Perspektiven ein. Für einen großen Teil der schwulen Bewegung spielten queere/intersektionale Debatten allerdings lange Zeit kaum eine Rolle. ${ }^{130}$ Im Vergleich dazu hat es in der lesbisch-feministischen Bewegung schon früh Interventionen, vor allem von migrantischen, jüdischen und Schwarzen Personen, aber auch aus der »KrüppelBewegung«, gegeben, die das homogene lesbische politische Subjekt in Frage stellten. Während die Gründung von LesMigraS bewegungsgeschichtlich in solchen Debatten zu

Ebd., S. $161 f$.

Wolfgang Förster/Tobias Natter/Ines Rieder: Der andere Blick: Lesbischwules Leben in Österreich. Eine Kulturgeschichte, Wien: MA 57 - Frauenförderung und Koordination von Frauenangelegenheiten 2001, S. $227 f$.

Helga Pankratz: »Österreich ist nicht das Paradies, aber ... «, in: an.schläge (2004).

Bis 1969 galt der in der NS-Zeit verschärfte $\S 175$, nach dem gleichgeschlechtliche sexuelle Handlungen unter Strafe gestellt wurden. Ab 1969 war gleichgeschlechtliche Sexualität ab einem Alter von 21 Jahren erlaubt.

S. Haunss: Identität in Bewegung, S. 194.

S. Hark: Queer Studies, S. 191f.

S. Hark: Queer Intervention; S. Hark: Vom Subjekt zur Subjektivität: Feminismus und die Zerstreuung des Subjekts.

Micheler, Stefan/Michelsen, Jakob: »Von der sschwulen Ahnengalerieく zur Queer Theory. Geschichtsforschung und Identitätsstiftung «, in: Ulf Heidel/Stefan Micheler/Elisabeth Tuider (Hg.), Jenseits der Geschlechtergrenzen. Sexualitäten, Identitäten und Körper in Perspektiven von Queer Studies, Hamburg: Männerschwarm Verlag 2001, S. 127-144. 
verorten ist, geht TransInterQueer e.V. wohl eher auf die sich ab den 1980er-Jahren formierenden Trans*"-Bewegungen zurück. Das Jugendnetzwerk Lambda BB hingegen ist noch in der DDR gegründet worden.

\section{Die Schwulenbewegung}

In der sich Anfang der 1970er-Jahre formierenden Schwulenbewegung standen sich zunächst politisch liberale konservative Gruppen und linke studentisch-marxistische Gruppen gegenüber. Zentrale und auch umkämpfte Themen der Schwulenbewegung waren die Abschaffung des Paragraphen 175, die Frage des Schutzalters, Pädophilie, die Ehe, der Umgang mit der Aids-Krise und die Verfolgung Homosexueller im Nationalsozialismus. ${ }^{131}$ Während vor allem liberale konservative Gruppen Teil einer stetig wachsenden kommerziellen Subkultur wurden, war für letztere die Kritik an den Kapitalverhältnissen zentral. Linke, marxistische Gruppen wie die »Homosexuelle Aktion Westberlin«, die »Rote Zelle Schwul« und die »Gay Liberation Front« eigneten sich nicht nur die Namen, sondern auch die out and proud-Politiken der schwulen Befreiungsbewegung in den USA an. ${ }^{132}$ Viele dieser radikaleren linken schwulen Zusammenschlüsse waren im Anschluss an den bekannten Film von Rosa von Praunheim - »Nicht der Homosexuelle ist pervers, sondern die Situation, in der er lebt « - gegründet worden, der auch als deutsches »Stonewall« gilt. ${ }^{133}$ Im sogenannten »Tuntenstreit « ${ }^{134} 1973$ trafen die unterschiedlichen Positionen der Schwulenbewegung aufeinander. Es ging vor allem darum, wer und welche politischen Strategien Teil der Schwulenbewegungen sein sollten. Als politischer Wendepunkt gilt das 1979 im Frankfurt stattfindende »Homolulu Festival«, das das Ende einer radikalen Schwulenbewegung mit "großen Idealen « markierte. ${ }^{135}$ In den 8oern hat sich die Schwulenbewegung dann stabilisiert beziehungsweise wird von Stagnation und Entpolitisierung der Bewegung gesprochen.

\section{Die Aids-Krise}

Das Thema Aids ist in Deutschland erst zwei Jahre später als in den USA aufgetaucht. Die schwule Bewegung reagierte vergleichsweise spät und verhalten darauf. ${ }^{136}$ Der Aktivist Andreas Salmen spricht von einer Kette der »Verharmlosung« in der Schwulenbewegung. ${ }^{137}$ Das lag vor allem daran, dass viele Schwule die Regulierung ihrer Sexualität durch Aids-Präventionskampagnen, in denen Polygamie und Promiskuität als Risikofaktor gesetzt wurden, ablehnten. Safer-Sex-Kampagnen wurden zum Teil als drastische Regulierung schwuler Sexualität und damit Identität gelesen. ${ }^{138}$ Die Frage nach

\footnotetext{
131 Vgl. P. Henze: Schwule Emanzipation und ihre Konflikte; S. Haunss: Identität in Bewegung.

132 S. Haunss: Identität in Bewegung, S. 194.

133 P. Henze: Schwule Emanzipation und ihre Konflikte, S. 182.

134 Der »Tuntenstreit« kam 1973 auf einer gemeinsamen Demo ins Rollen, als die Organisator*innen überlegten, die »Tunten«, die sich in ihrer Buntheit der gemeinsamen politischen Linie widersetzten, von der Demo auszuschließen.

135 P. Henze: Schwule Emanzipation und ihre Konflikte, S. 344.

136 S. Haunss: Identität in Bewegung, S. 192.

137 Jörg Hutter: »Schwule Konfliktunfähigkeit im Zeichen von Aids«, in: Vorgänge 122 (1993).

138 S. Haunss: Identität in Bewegung, S. 231.
} 
der Reaktion auf Aids wurde in der Schwulenbewegung zu einer umkämpften Identitätsfrage, auf die es nur zwei mögliche Antworten gab: entweder eine Politik der Anpassung oder das Ignorieren von Aids, um die auf sexuelle Freiheit ausgerichtete Identität aufrechterhalten. Neben der anfänglichen Verharmlosung des Themas entstanden von 1983 bis 1986 aber auch vermehrt Beratungsstellen und Hilfsangebote, an deren Aufbau schwule Aktivist*innen beteiligt waren. Die Angebote konnten auf staatliche Unterstützung zurückgreifen, da, ähnlich wie in Österreich, der deutsche Staat froh war, dass szenenahe Gruppen die Verantwortung für den Umgang mit Aids übernahmen. ${ }^{139}$ Viele schwule Aktivist*innen zogen sich aber schnell aus den institutionalisierten Angeboten zurück, da diese zu wenig politische Position bezogen. ${ }^{140}$

Ab 1985/86 konnte die Bedrohung durch Aids in der schwulen Community nicht mehr verleugnet werden. Dies führte dazu, dass zum einen vermehrt Selbsthilfegruppen von HIV-positiven Menschen ins Leben gerufen wurden und zum anderen eine Gleichstellung schwuler Lebensweisen gefordert wurde. Diese Politiken sind zentral verknüpft mit der Forderung nach der »Ehe für alle«, die mit dem Tod konfrontierten Menschen bestimmte Rechte einbringen sollte. Einzelne Aktivist*innen, wie Andreas Salmen und Rosa von Praunheim, hatten versucht, die Schwulenbewegung im Kontext von Aids zu politisieren, indem sie unter anderem nach amerikanischem Vorbild 1989 eine deutsche »Act Up«-Gruppe gründeten. Es gelang den deutschen »Act Up«Gruppen aber nicht, zu mobilisieren und politische Wirkung zu entfalten. ${ }^{141}$ Insgesamt - so Jörg Hutter - hat die deutsche Schwulenbewegung die Dimensionen der AidsEpidemie ignoriert. ${ }^{142}$ Sie schaffte es nicht, Aids als eigenes Politikfeld anzuerkennen, und verwies lieber auf die Zuständigkeiten der Aids-Organisationen. Gleichzeit trug die Aids-Krise dazu bei, die Sichtbarkeit von männlicher Homosexualität zu erhöhen, und gilt zumindest als Katalysator für die Akzeptanz einer (weißen, Mittelklasse-)Homosexualität. ${ }^{143}$

\section{Die Lesbenbewegung}

Lesbische Frauen waren in den Diskursen um Aids in zweifacher Hinsicht unsichtbar. Einerseits wurde ihnen eine monogame Lebensweise und damit kein Risikopotential zugeschrieben, andererseits wurde lesbischen Frauen keine aktive Sexualität zuerkannt. Dementgegen versuchten sie, die Aids-Krise und Safer-Sex-Themen zu nutzen, um die Unsichtbarmachung und Tabuisierung weiblicher Sexualität aufzubrechen. ${ }^{144}$

139 Ebd., S. 206.

140 So entstand in dieser Zeit eine Trennung zwischen denjenigen Schwulen, die HIV-positiv waren und die Angebote in Anspruch nahmen, und jenen, die ihre Sexualität »frei« und »unreguliert« ausleben wollten.

141 S. Haunss: Identität in Bewegung, S. 207f. Das hing, neben den ideologischen Debatten, wohl auch damit zusammen, dass der deutsche Staat Aids-Hilfen förderte und es in Deutschland, ähnlich wie in Österreich, eine funktionierende Cesundheitsversorgung gab.

142 J. Hutter: Schwule Konfliktunfähigkeit im Zeichen von Aids.

143 S. Haunss: Identität in Bewegung, S. 205.

144 Regina Brunett/Finn Jagow: »Macht und Homosexualität im Zeitalter von AIDS. AIDS als Knotenpunkt von Normalisierung und Selbstnormalisierung in Sexualitäten von Lesben und Schwulen«, in: Ulf Heidel/Stefan Micheler/Elisabeth Tuider (Hg.), Jenseits der Ceschlechtergrenzen. Sexualitä- 
Allerdings ist die Lesbenbewegung in der BRD weniger in der Schwulenbewegung, sondern in der autonomen Frauenbewegung zu verorten. ${ }^{145}$ Das "Lesbische Aktionszentrum « und das »Spinnenbodenarchiv« in Berlin gehörten zu den ersten lesbischen Räumen. Ansonsten nutzten lesbische Gruppen zumeist die Infrastruktur der Frauenbewegung. Auch thematisch stellte die Bezugnahme auf die Selbstbestimmung des eigenen Körpers und der Sexualität in der Frauenbewegung wichtige Anknüpfungspunkte für den lesbischen Aktivismus dar. Die Lesbenbewegung organisierte sich aber auch selbst (z.B. im Lesbenfrühlingstreffen) und schuf mit Zeitschriften wie der »UKZ (Unsere Kleine Zeitung)« (1974-2004), dem »Lesbenstich« (1980-1993) und dem »Ihrsinn« (1990-2004) eigene Gegenöffentlichkeiten. ${ }^{146}$ Bereits 1974 wurden amerikanische Debatten in einer Art und Weise (fehl-)angeeignet, dass statt von »Feminismus als Theorie, und Lesbischsein als Praxis«bald die Rede von der richtigen feministischen Revolution war, in der alle Frauen lesbisch sind. ${ }^{147}$ Oft galt das eindeutige lesbische revolutionäre Subjekt in den Anfängen der Lesbenbewegung als zentraler Ausgangspunkt lesbischer Politiken. ${ }^{148}$ Doch schon in den 8oer-Jahren wurde das homogene lesbische politische Subjekt vor allem durch kritische Interventionen von lesbischen Jüd*innen, Schwarzen Lesben und lesbischen Migrant*innen hinterfragt.

\section{Rassismus und Antisemitismus in der Lesbenbewegung}

Bereits 1980 gründeten in Berlin lebende lesbische Migrant*innen eine eigene Arbeitsgruppe auf einem Kongress in Frankfurt, und 1981 entstand in Berlin eine Frauengruppe, die sich mit Rassismus und Antisemitismus innerhalb und außerhalb der Frauenbewegung auseinandersetzte; ${ }^{149} 1984$ entstand mit der lesbisch-feministischen Gruppe »Schabbeskreis« der erste Zusammenschluss von lesbisch-jüdischen Frauen. Diese kritisierten unter anderem den unkritischen Umgang der Frauenbewegung mit dem Nationalsozialismus: Frauen wurden nur als Opfer des patriarchalen Systems, nicht aber als Täter*innen gedacht. Auch Schwarze, afrodeutsche (lesbische) Frauen schlossen sich $1986 \mathrm{zu}$ der bis heute bestehenden Gruppe "Adefra« zusammen. ${ }^{150}$ Die Konflikte um Rassismus und Antisemitismus spitzten sich teilweise so weit $\mathrm{zu}$, dass beispielsweise die bloße Anwesenheit des "Schabbeskreises « als Provokation galt. ${ }^{151}$ Podiumsdiskussionen wurden abgebrochen und die Verantwortung für die Bekämpfung

ten, Identitäten und Körper in Perspektiven von Queer Studies, Hamburg: Männerschwarm Verlag 2001, S. 190-206, hier S. 202.

145 P. Henze: Schwule Emanzipation und ihre Konflikte, S. $327 f$.

146 Agnes S. Münst: »Lesbenbewegung: Feministische Räume positiver Selbstverortung und gesellschaftlicher Kritik«, in: Ruth Becker/Beate Kortendiek (Hg.), Handbuch Frauen- und Geschlechterforschung, Wiesbaden: Springer Verlag 2010, S. 692-697, hier S. $905 f f$.

147 Sabine Hark: Vom Subjekt zur Subjektivität: Feminismus und die Zerstreuung des Subjekts (= Berliner Wissenschaftlerinnen stellen sich vor) 1992, S. 13.

148 Vgl. Sabine Hark: »Queer Intervention«, in: Madeleine Marti/Angelika Schneider/Irena Sgier et al. (Hg.), Querfeldein. Beiträge zur Lesbenforschung, Bern: eFeF-Verlag 1994, S. 210-221.

149 C. Leidinger: Frühe Debatten um Rassismus und Antisemitismus in der (Frauen- und) Lesbenbewegung in den 1980er Jahren der BRD, S. 24.

150 Ebd., S. 26f.

151 Ebd., S. 26ff. 
von Rassismus und Antisemitismus wurde den betroffenen Frauen zugeschrieben. ${ }^{152}$ Politische Bündnisse waren zunächst kaum möglich und viele jüdische und Schwarze Frauen zogen sich zurück und organisierten sich selbstständig. Nicht zuletzt hinterfragten migrantische und Schwarze Frauen auch den Begriff »Lesbe« als westliches Konzept, durch das sie sich nicht repräsentiert sahen. ${ }^{153}$ Trotz anfänglicher Ignoranz und vielfältiger Konflikte fand Ende der 8oer-Jahre zunehmend eine Sensibilisierung in der Frauen- und Lesbenbewegung für Rassismus und Antisemitismus statt, die das homogene lesbische Wir zum Bröckeln brachte. Dies spiegelt sich auch in dem Motto der »Lesbenwoche« 1988 - »Abgrenzung, Ausgrenzung, Begrenzung« - wider, auf der es erste Versuche gab, Bündnisse mit Schwarzen, jüdischen und migrantischen Lesben sowie »Krüppellesben« zu schließen. ${ }^{154}$ Vier Jahre später ist in der Berliner Lesbenberatung ein eigener Arbeitsschwerpunkt zur Schnittstelle von Rassismus, Geschlecht und Sexualität entstanden, aus dem 1999 LesMigraS - eines der im Zentrum der vorliegenden Untersuchung stehenden Projekte - hervorgegangen ist.

\section{Trans*-Aktivismus}

Seit den 1970er-Jahren organisieren sich in Deutschland auch Trans*-Gruppen. Zunächst auf lokaler Ebene, wie beispielsweise eine 1972 gegründete Gruppe, die sich für die rechtliche Anerkennung des Vornamens und Geschlechts einsetzte. Ab Mitte der 8oer-Jahre entstand auf überregionaler Ebene dann mit »Transidentas e.V.« die erste Trans*-Gruppe. Ab den 1990er-Jahren konsolidierte sich der Trans*-Aktivismus in Deutschland. Dabei bildeten sich zwei unterschiedliche Strömungen heraus: zum einen jene Gruppen, die eine queer-theoretische Perspektive vertreten, und auf der anderen Seite solche, die sich auf neurobiologische Grundlagen berufen. Gemeinsam ist ihnen jedoch der Kampf für mehr Selbstbestimmung und gegen Diskriminierung. ${ }^{155}$ Die Forderungen nach dem Recht auf Selbstbestimmung über den eigenen Körper und eine queere Haltung verbindet im 2004 gegründeten Verein TransInterQueer e.V. - ebenfalls eine der im Zentrum dieser Arbeit stehenden Einrichtungen - auch Trans* - und Inter*-Themen. Die Zusammenarbeit von Trans*- und Inter*156 -Personen unter einem Dach ist eine für den deutschsprachigen Raum besondere Konstellation.

152 Ebd., S. 24-28.

153 Maria do Mar Castro Varela/Encarnación Gutiérrez Rodríguez: »Queer Politics im Exil und in der Migration«, in: Nico ]. Beger/Sabine Hark/Antke Engel et al. (Hg.), Queering-Demokratie. Sexuelle Politiken, Berlin: Querverlag 2000, S. 100-112, hier S. 110.

154 C. Leidinger: Frühe Debatten um Rassismus und Antisemitismus in der (Frauen- und) Lesbenbewegung in den 1980 er Jahren der BRD, S. 28.

155 Adrian de Silva: „Grundzüge struktureller und konzeptueller Entwicklungen der Trans*bewegung in der Bundesrepublik Deutschland seit Ende der 1990er Jahre«, in: Bundesstiftung Magnus Hirschfeld (Hg.), Forschung im Queerformat. Aktuelle Beiträge der LSBTI*-, Queer- und Ceschlechterforschung, Bielefeld: transcript Verlag 2014, S. 151-171, hier S. $151 \mathrm{f}$.

156 Viele Inter*-Gruppen können auch aufgrund eines biologistischen Zugangs zum Körper oft nichts mit queer anfangen. Andere kritisieren eine Vereinnahmung durch queer oder sehen viele Menschen ihrer Gruppe nicht durch den Begriff repräsentiert. 


\section{Die Schwulen- und Lesbenbewegung in der DDR}

Die Situation von Schwulen und Lesben in der DDR war eine andere als in der BRD. Auf der einen Seite galt »die Familie als kleinste Zelle der sozialistischen Gesellschaft«, auf der anderen Seite war Sexualität in den Bereich des Privaten verschoben, in dem grundsätzlich alles erlaubt war. ${ }^{157}$ Geschlechterthemen galten zudem als Nebenwiderspruch, der sich mit dem Hauptwiderspruch auflösen sollte. Für Schwule und Lesben war es lange Zeit schwer, sich zu engagieren, weil sie im Verdacht standen, das Regime möglicherweise in Frage zu stellen. ${ }^{158}$ Trotzdem gab es bereits seit 1973 erste schwul-lesbische Zusammenschlüsse und 1986 wurde der bis heute bestehende »Sonntagsclub« gegründet. Es formierten sich in den nächsten Jahren immer mehr Gruppen, die gut untereinander vernetzt waren, und ab 1987 kann von einer Lesbenbewegung in der DDR gesprochen werden. ${ }^{159}$ Grundsätzlich gab es in der DDR auf der einen Seite »weltliche« Zusammenschlüsse, die für sich eine Integration von Homosexualität in die Normalität des Realsozialismus einsetzten, und solche, die unter dem Dach der Kirche vielfach auch regimekritisch waren. ${ }^{160}$ Jene Gruppen, die den Raum der evangelischen Kirche nutzten, um sich zusammenzuschließen, waren von Anfang an auch eng mit der Friedens- und der Bürgerrechtsbewegung verknüpft, die die gleichen Orte nutzten. ${ }^{161}$ Daher setzten diese Aktivist*innen sich bereits früh auch mit den Interventionen Schwarzer Lesbischer Frauen auseinander. ${ }^{162}$ So geht auch die Idee des Runden Tischs auf die Bürgerrechtsbewegung zurück. ${ }^{163}$ Das Jugendnetzwerk BB - das dritte deutsche Projekt, das für die folgende Untersuchung ausgewählt wurde - ist an diesem sogenannten Runden Tisch in der DDR gegründet worden, an den von Dezember 1989 bis März 1990 regelmäßig Vertreter*innen geladen waren, um Geschlechterinteressen gemeinsam zu verhandeln.

\subsubsection{Schweiz}

Während die deutschen und österreichischen schwul-lesbischen Strukturen nach dem Nationalsozialismus wiederaufgebaut werden mussten, existierten in der Schweiz kontinuierlich Gruppen, Clubs und eigene Zeitschriften. In den 3oer-Jahren gab es in der

157 Ursula Silge: »Damals war's. Ein Rückblick auf Bedingungen und Strukturen der lesbisch-schwulen Bewegung in der DDR«, in: Cabriele Dennert/Christiane Leidinger/Franziska Rauchut (Hg.), In Bewegung bleiben. 100 Jahre Politik, Kultur und Geschichte von Lesben, Berlin: Querverlag 2007, S. 105-108, hier S. 105.

158 Ebd.

159 Gabriele Dennert/Christiane Leidinger/Franziska Rauchut: »Wir sind keine Utopistinnen<. Lesben in der DDR«, in: Gabriele Dennert/Christiane Leidinger/Franziska Rauchut (Hg.), In Bewegung bleiben. 100 Jahre Politik, Kultur und Geschichte von Lesben, Berlin: Querverlag 2007, S. 95-104, hier S. $98 \mathrm{f}$.

160 Ebd., S. $97 f$.

161 Ebd., S. 99.

162 Marina Krug: »Die Gruppe Arbeitskreis Homosexuelle Selbsthilfe. Lesben in der Kirche in Berlin/DDR - November 1982 bis Sommer 1986«, in: Gabriele Dennert/Christiane Leidinger/Franziska Rauchut (Hg.), In Bewegung bleiben. 100 Jahre Politik, Kultur und Geschichte von Lesben, Berlin: Querverlag 2007, hier S. 110.

163 G. Dennert/C. Leidinger/F. Rauchut: >Wir sind keine Utopistinnen<, S. 101. 
Schweiz noch viele Räume schwul-lesbischer Zusammenarbeit. Diese nahmen beginnend mit der Gründung der schwulen Organisation »Der Kreis ${ }^{164} 1943$ langsam ein Ende. ${ }^{165}$

Wann und wie »queer« in der Schweiz Einzug gehalten hat, ist kaum aufgearbeitet. Der vom schweizerischen lesbischen Verein »Sappho« 1994 herausgegebene Sammelband "Queerfeld«, der im Anschluss an das zweite Symposium deutschsprachiger Lesbenforschung 1994 veröffentlicht wurde, verweist jedoch zum einen auf eine Vernetzung mit der deutschsprachigen Lesbenbewegung ${ }^{166}$ und zum anderen lassen auch von Schweizer Aktivist*innen veröffentlichte Artikel bereits queere Perspektiven erkennen. ${ }^{167}$ Auch in der Schweiz ist »queer « nicht aus einer politischen Notwendigkeit heraus entstanden, sondern aus anderen Ländern übernommen worden.

Die Milchjugend ist in einer Zeit entstanden, in der nicht nur »queer« in der Schweiz kaum eine Rolle spielte, sondern auch die Organisation von Jugendgruppen selten geworden war. Während es in den 1980er-Jahren noch recht viele Jugendgruppen in der Schweiz gegeben hatte, gab es zur Zeit der Gründung der Milchjugend kaum noch welche. ${ }^{168}$ Allerdings konnte sich die Milchjugend die schwul-lesbischen Strukturen in der Schweiz zu Nutze machen. Die Jugendlichen wurden von Anfang an von Organisationen wie der »Aids-Hilfe«, den »homosexuellen Arbeitsgruppen«, »Pink Cross« und »LOS« unterstützt, mit denen die Einrichtung bis heute eng zusammenarbeitet.

\section{Die Schwulenbewegung}

Vor allem in den 5oer- und 6oer-Jahren war die homosexuelle Szene in der Schweiz trotz Entkriminalisierung von Homosexualität ab einem Schutzalter von zwanzig Jahren im Jahr 1942 - homophober Gewalt und Schikane von Seiten des Staates und der Medien ausgesetzt. ${ }^{169} 1966$ wurde das von schwulen Männern geführte Lokal »ContiClub« in Zürich eröffnet, das 1968 vom »Club $68(\mathrm{SOH}) \ll$ übernommen wurde. In diesem

164 Die von 1943 bis 1965 bestehende Gruppe war hauptsächlich um die international bekannte und anerkannte Zeitschrift »Der Kreis« angeordnet. Die Zeitschrift wurde nicht zuletzt aufgrund ihrer Mehrsprachigkeit und ihres literarischen Gehalts, aber auch des Nationalsozialismus wegen in angrenzenden Staaten viel gelesen. Die Gruppe ist ebenfalls bekannt für die alljährlichen Neujahrsbälle, zu denen Schwule auch von außerhalb der Schweiz in Scharen strömten. Diese Neujahrsbälle sind von der Milchjugend als queere Neujahrsbälle wieder ins Leben gerufen worden. Die Milchjugend pflegt bis heute regelmäßigen Kontakt zu einem ehemaligen Mitglied des »Kreis«. B. Gerber: Lila ist die Farbe des Regenbogens, Schwestern, die Farbe der Befreiung ist rot: die homosexuellen Arbeitsgruppen in der Schweiz (HACH) von 1974-1995, S. 27.

165 Ebd.

166 Sabine Hark hat an dem Symposium teilgenommen und veröffentlichte in eben jenem Band den Aufsatz »Queer Interventionen«.

167 »Es geht nicht nur um die Rekonstruktion der Lebenszusammenhänge lesbischer Frauen, sondern auch um die Dekonstruktion von Heterosexualität als normierende Struktur.« Madeleine Marti/Angelika Schneider/Irena Sgier et al. (Hg.): Querfeldein. Beiträge zur Lesbenforschung, Bern: eFeF-Verlag 1994, S. 65. Hinweis Cespräch mit Corinne Rufli.

169 Christa Wirth/Anja Suter: Unverschämt. Lesben und Schwule gestern und heute 2003, S. 46. Es gab regelmäßig Polizeirazzien, Schwulenregister und Morde an Schwulen, die medial inszeniert wurden und rechtlich kaum Konsequenzen hatten. 
zunächst männlich geführten Club trafen sich ab 1969 auch erstmals (wieder) lesbische Frauen, die später auch Mitglieder im »Club 68« wurden. ${ }^{170}$ Der »SOH (Schwule Organisation Homophiler)« war eine eher konservative schwule Organisation, die jahrelang in Abgrenzung zu, aber auch in Zusammenarbeit mit »radikaleren« Gruppen agierte. Insgesamt waren die Politiken der »SOH« gemäßigte Versuche, in die Normalität integriert zu werden. Sie wollten keine umfassende Revolution und vermieden es aufzufallen. Allerdings rezipierte die »SOH« in ihrer Zeitschrift »Club 68« schon 1969 Artikel zu den Ereignissen in den USA, denen die Gruppe allerdings kritisch gegenüberstand. Von den eher konservativen Politiken grenzten sich die in den 7oer-Jahren entstehenden "Homosexuellen Arbeitskreise « ab. ${ }^{171}$ Sie sind vor allem im Zuge der Student"innenbewegungen in mehreren Städten in der Schweiz entstanden. Katalysator war, ähnlich wie in Deutschland, die Vorführungen des Rosa-von-Praunheim-Films. Sie grenzten sich von den konservativeren Gruppen insofern ab, als sie nicht für brave Anpassungspolitik standen. Das drückte sich auch in der Ablehnung der Selbstbezeichnung »homophil« aus, die durch ein »stolzes« Selbstverständnis als schwul oder homosexuell ersetzt wurde. Die Homosexuellenarbeitsgruppen und insbesondere der Dachverband »HACH Homosexuelle Arbeitsgruppe Schweiz « waren politisch links-marxistisch ausgerichtet. ${ }^{172}$ Zentrale politische Ziele der »HACH« waren Forderungen nach gleichen Rechten beziehungsweise den Privilegien der Ehe, ${ }^{173}$ die Revision des Sexualstrafrechts ${ }^{174}$ und die Abschaffung der Schwulenkarteien. ${ }^{175} 1978$ wurde bereits in Zusammenarbeit mit der »konservativeren « schwulen Gruppe »SOH« und der »Homosexuellen Frauengruppe Zürich (HFG)« die erste Erinnerung an die »Stonewall Inn«-Proteste organisiert. Ein Jahr später fand die erste Pride-Demonstration in Bern statt. ${ }^{176}$ In den späten 7oerund 8oer-Jahren wurden zahlreiche weitere schwule Gruppen ins Leben gerufen und die Bewegung differenzierte sich entsprechend aus. Ab Mitte der 8oer-Jahre formierten sich auch einige homosexuelle Jugendgruppen wie beispielsweise "Albatros« in St. Gallen, »SPOIZ« in Bern und »Rose« (später »Grenzenlos«) in Basel, die sozusagen als Vorläufer der Milchjugend gelten können. ${ }^{177}$

170 Das Wiki zur Lesbengeschichte der Schweiz: Conti-Club, https://l-wiki.ch/Conti-Club.

171 Ausgehend von der 1971 gegründeten »Homosexuellen Arbeitsgruppe Zürich « entstanden in den nächsten vier Jahren die »Homosexuelle Arbeitsgruppe Basel (HABS) «, »Bern (HAB) «und »St. Gallen (HASC) 1973 begannen die vier Arbeitsgruppen, die übergeordnete »Homosexuelle Arbeitsgruppe Schweiz $(\mathrm{HACH})$ « zu gründen, in der Informationen ausgetauscht und gebündelt werden sollten.

172 B. Gerber: Lila ist die Farbe des Regenbogens, Schwestern, die Farbe der Befreiung ist rot: die homosexuellen Arbeitsgruppen in der Schweiz (HACH) von 1974-1995, S. 35-66.

173 Ebd., S. $101 \mathrm{ff}$.

174 Ebd., S. 78.

175 Ebd., S. 107.

176 Ebd., S. $110 f$.

177 Ernst Ostertag/Röbi Rapp: Schwulengeschichte, https://schwulengeschichte.ch/home/ vom 15.09. 2020. 


\section{Die Aids-Krise}

Als 1983 die Aids-Krise auch in der Schweiz zum Thema wurde, reagierten die »HACH« und die »HA-Gruppen« schnell in Form von Briefkastenaktionen, Informationsarbeit, Interventionen und Flugblättern. Der » $\mathrm{HACH}$ « war es von Anfang an wichtig, Aids nicht nur als eine Gesundheitskrise, sondern als Politikum zu verstehen. Entsprechend versuchte sie gleichzeitig medizinische Aufklärungsarbeit zu leisteten, gegen die negative Berichterstattung zu protestieren und die Politik in die Verantwortung zu nehmen. In und um die »HACH« formierte sich relativ schnell die Arbeitsgruppe »Schwule Mediziner«, die gemeinsam mit zahlreichen anderen Organisationen an der Begründung der Aids-Hilfe Schweiz im Jahr 1985 beteiligt war. Auch in der Schweiz lösten die Versuche, mit einer Regulierung von Sexualität auf die Aids-Krise zu antworten, Kontroversen aus. Aufgrund von Konflikten um den mangelnden politischen Gehalt der Aids-Hilfe zog die »HACH « sich schnell wieder aus ebendieser zurück. Viele andere schwule Gruppen blieben aber Teil der Aids-Hilfe. Durch die Aids-Krise kam es auch in der Schweiz vermehrt zu einer Institutionalisierung und Professionalisierung der Bewegung. ${ }^{178} \mathrm{Im}$ Zuge der Forderung von schwulen Gruppen - unter anderem der »HACH« - nach bezahlten Stellen für die Schwulenarbeit ist 1993 der landesweite Verband »Pink Cross« ins Leben gerufen worden, der bis heute eine wichtige Interessenvertretung für Schwule in der Schweiz ist. ${ }^{179}$ Sowohl die Aids-Hilfe als auch »Pink Cross « und verschiedene homosexuelle Arbeitsgruppen haben von Anfang an die Milchjugend unterstützt. ${ }^{180}$

\section{Die Lesbenbewegung}

Lesbische Frauen hatten weniger mit Razzien und Schwulenkarteien zu kämpfen, sondern damit, dass es für Frauen in der Schweiz lange wenig Möglichkeiten für eine persönliche und berufliche Unabhängigkeit gab. Auf nationaler Ebene wurde das Frauenwahlrecht erst 1971 eingeführt und es wurde erst 1990 auch im letzten Kanton umgesetzt. ${ }^{181}$ Die homosexuellen Arbeitsgruppen waren grundsätzlich für Frauen offen, blieben zumeist aber männlich dominiert. So berichtet eine Aktivist* in aus der Ostschweiz, dass sie in St. Gallen lange Zeit die einzige Frau in der homosexuellen Arbeitsgruppe gewesen sei und die Gruppe verlassen habe, nachdem schwule Männer geäußert hatten, dass sie sich nicht auch noch mit Frauenproblemen herumschlagen wollten. ${ }^{182}$ Gegen solche Ausgrenzungen wurde die »Homosexuelle Frauengruppe (HFG) St. Gallen« ins Leben gerufen, die ähnlich wie die damals bereits bestehende »HFG Zürich«an

178 B. Gerber: Lila ist die Farbe des Regenbogens, Schwestern, die Farbe der Befreiung ist rot: die homosexuellen Arbeitsgruppen in der Schweiz (HACH) von 1974-1995, S. 116-122.

179 Während die »HACH« sich einige Zeit später aufgelöste, bestehen viele der damals gegründeten Arbeitsgruppen sowie andere später gegründete Gruppen unter dem Dachverband von »Pink Cross« immer noch. Aktuell sind 38 Vereine Mitglied von »Pink Cross«.

180 Milchjugend: Wir machen Druck, Milchbüechli 2012.

181 Francois Baur/Alecs Recher: »Historische Entwicklung«, in: Andreas R. Ziegler/Michel Montini/Eylem A. Copur (Hg.), LGBT-Recht. Rechte der Lesben, Schwulen, Bisexuellen und Transgender in der Schweiz, Basel: Helbing Lichtenhahn 2015, S. 1-42, hier S. $16 \mathrm{f}$.

182 Myrjam Cabernad: »Lesbenbewegung in der Ostschweiz - Spurensuche in der Provinz«, in: Doris Brodbeck (Hg.), Neue Frauenbewegung, St. Gallen: Sabon-Verlag 2005, S. 45-54, hier S. $48 f$. 
die Frauenbewegung angebunden war. Die »HFG Zürich « - die erste lesbische Frauengruppe der 70er-Jahre - entstand 1974 in Abspaltung zur »HAZ (Homosexuelle Arbeitsgruppe Zürich)«. In der ersten Ausgabe der »Lesbenfront« begründeten die Frauen die Trennung folgendermaßen: »Wir Lesben haben eingesehen, dass eine Zusammenarbeit mit den Phallokraten der $>\mathrm{HAZ}$ « unmöglich ist. « ${ }^{183}$ Zunächst war die »HFG« allerdings mit der Schwierigkeit konfrontiert, dass zum einen viele Frauen eher Interesse hatten, andere Frauen kennenzulernen, als sich politisch zu engagieren, und dass zum anderen wenige Frauen öffentlich geoutet waren. ${ }^{184}$

Nachdem sich aber ab 1975 die »Radikal-feministische Lesbengruppe« als Arbeitsgruppe formierte, kam Bewegung in die »HFG « und es entstanden viele neue unterschiedliche Arbeitsgruppen. Im gleichen Jahr wurde auch die erste Ausgabe der »Lesbenfront « veröffentlicht. Die »HFG« nutzte die Räume des Frauenzentrums von Beginn $a^{185}$ und war auch über die Nutzung der räumlichen Infrastrukturen hinaus in der Frauenbewegung verortet. ${ }^{186} 1976$ organisierte sich die »HFG « als eigener Verein, die Nähe zur Frauenbewegung blieb aber bestehen. ${ }^{187}$ Der thematischen Ausrichtung der »Lesbenfront « zufolge spielte auch die Auslotung eines stolzen lesbischen Selbstverständnisses eine wichtige Rolle für die Lesbenbewegung. Weitere wichtige Themen in der Lesbenfront waren Sexualität, Mutterschaft, Sexismus und Gewalt sowie das zum Teil konflikthafte Bündnis mit heterosexuellen Frauen. Neben der eigenen Zeitschrift organisierte die »HFG« auch nationale Lesbentreffen und andere Orte des Austauschs wie Theater oder Discos. Auch in anderen Städten entstanden in den nächsten Jahren, oft in Abspaltung von den »Homosexuellen Arbeitsgruppen«, lesbische Gruppen wie die 1977 in Bern gegründete Gruppe »LIB (Lesben Initiative Bern)« oder die ebenfalls 1977 gegründete, bereits erwähnte »HFG St. Gallen «. ${ }^{188}$ In den $80 e r-J a h r e n$ sind viele weitere lesbische Frauengruppen in verschiedenen Städten in der Schweiz entstanden. ${ }^{189}$ Mit »LOS (Lesbenorganisation Schweiz)« ist in den 1990er-Jahren die erste nationale Lesbenorganisation ins Leben gerufen worden, die ähnlich wie »Pink-Cross« bis heute existiert und die Milchjugend seit ihren Anfängen unterstützt hat. ${ }^{190}$

183 HFG: Homosexuelle Frauengruppe Zürich. Lesbenfront 1975, HFC: Homosexuelle Frauengruppe Zürich. Lesbenfront 1975, https://www.e-periodica.ch/digbib/view?pid=les-001\%3A1975\%3A0\#5 vom 16.12.2020, S. 2

184 Corinne Rufli: Seit dieser Nacht war ich wie verzaubert. Frauenliebende Frauen über siebzig erzählen, Baden: Hier und Jetzt 2015, S. 145.

185 Das Wiki zur Lesbengeschichte der Schweiz.

186 Zunächst wurden sie auf Flugblättern als Arbeitsgruppe der Frauenbefreiungsbewegung bezeichnet und am 8. März 1974 gab es bereits den ersten lesbischen Block auf der Demonstration in Basel.

187 Homosexuelle Frauengruppe Zürich: Lesbenkampf ist Frauenkampf 1976, S. 8.

188 Das Wiki zur Lesbengeschichte der Schweiz.

189 Einen Überblick zu den verschiedenen Lesbenorganisationen in der Schweiz findet sich unter folgendem Link: https://l-wiki.ch/Kategorie:Organisation.

190 C. Caprez/Y. E. Nay: Frauenfreundschaften und lesbische Beziehungen, S. 242. 


\section{Trans*- und migrantischer Aktivismus}

Die erste Trans*-Gruppe - »Verein Femmes Travesties« - ist 1980 in Zürich gegründet worden. In den nächsten Jahren entstanden weitere Austauschräume wie die Gruppe »Girls Wanna Have Fun« (1995) oder das »Transensyndikat« (2000). Das erste regelmäßige Treffen für Trans*-Männer fand 2008 statt, und zwei Jahre später wurde die nationale Lobbyorganisation, Rechtsberatung und Interessenvertretung »Transgender Network Switzerland « ins Leben gerufen. ${ }^{191}$ Migrantische oder of Color sexual- und geschlechterpolitische Organisation gab es in der Schweiz bis dahin wenig. ${ }^{192}$ Auch der Begriff »queer« hat erst vergleichsweise spät in der Schweiz Einzug erhalten. Von 2008 bis 2017 gab es in der Schweiz das "Queere Filmfestival Basel« und auch das 2004 gegründete Netzwerk »Sündikat« versteht sich als »queer«. Ebenso können die innerhalb des Feminismus entstandenen Ladyfeste, die 2008 das erste Mal stattfanden, als queere Räume verortet werden. ${ }^{193}$ Die »Homosexuelle Arbeitsgruppe« in Basel benannte sich 2017 in »habsqueer Basel« um und will damit mehr Offenheit repräsentieren. Die »Romanescos« organisieren sich seit einigen Jahren als Treffpunkt für genderqueere*, nonbinary und andere Personen. ${ }^{194}$

191 F. Baur/A. Recher: Historische Entwicklung, S. $19 f$.

192 Bettina Büchler: »Alltagsräume queerer Migrantinnen in der Schweiz: ein Plädoyer für eine räumliche Perspektive auf Intersektionalität«, in: Christa Binswanger/Margaret Bridges/Brigitte Schnegg et al. (Hg.), Gender Scripts. Widerspenstige Aneignungen von Geschlechternormen, Frankfurt a.M.: Campus Verlag 2009, S. 41-61, hier S. 42.

193 Kristina Schulz/Leena Schmitter/Sarah Kiani: Frauenbewegung - Die Schweiz seit 1968. Analysen, Dokumente, Archive, Baden: Hier und Jetzt 2014, S. 146.

194 Romanescos: Über uns, https://www.romanescos.ch/Ueber-uns/ vom 28.11.2020. 
